\title{
Stochastic homogenization of the Keller-Segel chemotaxis system
}

\author{
Anastasios Matzavinos ${ }^{\mathrm{a}, \mathrm{b}, 1}$, Mariya Ptashnyk $\mathrm{k}^{\mathrm{c}, 2}$ \\ ${ }^{a}$ Division of Applied Mathematics, Brown University, Providence, RI 02912, USA \\ ${ }^{b}$ Computational Science and Engineering Laboratory, ETH Zürich, CH-8092, Zürich, Switzerland \\ ${ }^{c}$ Division of Mathematics, University of Dundee, Dundee, DD1 4HN, UK
}

\begin{abstract}
In this paper, we focus on the Keller-Segel chemotaxis system in a random heterogeneous domain. We assume that the corresponding diffusion and chemotaxis coefficients are given by stationary ergodic random fields and apply stochastic two-scale convergence methods to derive the homogenized macroscopic equations. In establishing our results, we also derive a priori estimates for the Keller-Segel system that rely only on the boundedness of the coefficients; in particular, no differentiability assumption on the diffusion and chemotaxis coefficients for the chemotactic species is required. Finally, we prove the convergence of a periodization procedure for approximating the homogenized macroscopic coefficients.
\end{abstract}

Keywords: Chemotaxis, stochastic homogenization, two-scale convergence, Palm measures, point processes.

\section{Introduction}

Chemotaxis as a term refers to the directed movement of cells and microorganisms in response to a chemical signal. Historically, the first mathematical model of chemotaxis was proposed by Keller and Segel in order to investigate the aggregation dynamics of cellular slime molds, such as the social amoeba Dictyostelium discoideum [30]. Since then, the Keller-Segel model has been analyzed extensively, and a comprehensive review of related mathematical results can be found in the two articles by Horstmann [23, 24].

It is well known that in one dimension the Keller-Segel model is well-posed globally in time. Global existence and boundedness of solutions in one dimension were first shown by Yagi [46] by means of energy estimates. Moreover, the well-posedness and the existence of a finite-dimensional attractor for the one-dimensional model was proved by Osaki and Yagi [41].

The dynamics of the Keller-Segel model in two and three dimensions are more complex than the one-dimensional case, since in higher dimensions the solutions may blow up in finite time $[27,38,45,47]$. Several results that appeared in the 1990's have demonstrated

\footnotetext{
${ }^{1}$ E-mail address: matzavinos@brown.edu

${ }^{2}$ E-mail address: m.ptashnyk@dundee.ac.uk Preprint submitted to Elsevier
} 
that in two and three dimensions the Keller-Segel model is well-posed globally in time for "small" initial data. However, in the presence of "large" initial data, the solutions blow up; in other words, they do not remain bounded [25, 26, 39, 46].

Corrias and Perthame [11] showed that in $d$ dimensions, the Keller-Segel model is critical in $L^{d / 2}$, which is to say that the "smallness" or "largeness" of the initial data is determined in terms of the $L^{d / 2}$ norm. Similar conditions were derived in $[12,13]$ for a parabolic-elliptic variation of the Keller-Segel model. The global behavior of a twodimensional parabolic-parabolic chemotaxis system, under the assumption of "small" initial data, was investigated by Gajewski and Zacharias [18].

As alluded to in the above paragraphs, there is a wealth of results on the existence and regularity of solutions of the Keller-Segel model. However, there is no literature investigating homogenization approaches and the influence of substrate heterogeneity on the dynamics of the model.

Stochastic homogenization is a growing field in multiscale analysis. Some of the first results on the stochastic homogenization of linear second-order elliptic equations were obtained by Kozlov [32] (by a direct contraction of the corrector functions), by Papanicolaou and Varadhan [43] (by using Tartar's energy method), and by Zhikov et al. [50] (by using $G$-convergence of operators). Subsequently, the homogenization of quasi-linear elliptic and parabolic equations with stochastic coefficients was considered by Bensoussan and Blankenship [6] and Castell [10]. The stochastic homogenization of convex integral operators by means of $\Gamma$-convergence was considered by Dal Maso and Modica [15, 16]. The method of viscosity solutions was employed by Caffarelli et al. [9] to derive effective equations for fully nonlinear elliptic and parabolic equations in stationary ergodic media. In a similar fashion, subadditive ergodic theory has been used together with the theory of viscosity solutions or variational representations of solutions and the minimax theorem to homogenize Hamilton-Jacobi and viscous Hamilton-Jacobi equations in stationary ergodic media [2, 31, 34, 35] (see also references therein). More recently, subadditive ergodic theory has also been employed to homogenize quasiconvex (level-set convex) and, more generally, non-convex Hamilton-Jacobi equations in stationary ergodic media $[3,4]$.

The theory of periodic two-scale convergence $[1,37,40]$ has been extended in the stochastic setting by Bourgeat, Mikelić, and Wright [8], who defined the concept of twoscale convergence in the mean, and by Zhikov and Piatnitski [52], who defined an explicitly stochastic two-scale convergence for random measures. The two-scale convergence in the mean has been applied to derive macroscopic equations for single- and two-phase fluid flows in randomly fissured media [7, 49]. The stochastic two-scale convergence has been extended to Riemannian manifolds and has been applied to analyze heat transfer through composite and polycrystalline materials with nonlinear conductivities [20,21].

The paper is organized as follows. In section 2, we formulate a microscopic chemotaxis model with diffusion and chemotaxis coefficients for the chemotactic species given by stationary ergodic random fields. In contrast, and consistent with the experimental setting discussed in section 2, the diffusion coefficient of the chemical species (chemoattractant) is assumed to be deterministic, i.e., independent of the random medium. We then derive a priori estimates in section 3 and prove the existence and uniqueness of weak solutions for the microscopic model. Our derivation of the a priori estimates differs from those found in [22], [39], or [41], as we only assume the boundedness of the rapidly oscillating coefficients describing the stochastic medium. In section 4 , we use the 
derived a priori estimates and the notion of stochastic two-scale convergence to derive a macroscopic (homogenized) model for our system. Two auxiliary stochastic problems are obtained to define the macroscopic diffusion and chemosensitivity coefficients for the chemotactic species. In section 5, we use a periodization procedure and prove the convergence of the effective coefficients obtained by periodic approximation to the corresponding macroscopic coefficients obtained by the stochastic homogenization approach of section 4 .

\section{Formulation of the problem}

We consider a variation of the original Keller-Segel model of chemotaxis [30], where the coefficients of the model are defined by stationary random fields. Specifically, we consider the system:

$$
\begin{aligned}
& u_{t}^{\varepsilon}=\nabla \cdot\left(D_{u}^{\varepsilon}(x) \nabla u^{\varepsilon}-\chi^{\varepsilon}(x) u^{\varepsilon} \nabla v^{\varepsilon}\right), \quad x \in Q, t>0, \\
& v_{t}^{\varepsilon}=\quad \nabla \cdot\left(D_{v}(x) \nabla v^{\varepsilon}\right)-\gamma v^{\varepsilon}+\alpha u^{\varepsilon}, \quad x \in Q, t>0, \\
& \frac{\partial u^{\varepsilon}}{\partial n}=0, \quad \frac{\partial v^{\varepsilon}}{\partial n}=0, \quad x \in \partial Q, t>0, \\
& u^{\varepsilon}(0, x)=u_{0}(x), \quad v^{\varepsilon}(0, x)=v_{0}(x), \quad x \in Q,
\end{aligned}
$$

where $Q \subset \mathbb{R}^{d}$ is a bounded domain and $\alpha, \gamma$ are positive constants. Moreover, $u^{\varepsilon}$ and $v^{\varepsilon}$ denote the density of a population of cells (the chemotactic species) and the concentration of a chemoattractant, respectively.

As will become apparent in the following, the parameter $\varepsilon$ represents the spatial scale of the microscopic structure of the underlying medium or substrate. The diffusion coefficient $D_{u}^{\varepsilon}$ and the chemosensitivity function $\chi^{\varepsilon}$ depend on $\varepsilon$, as they are affected by changes in the properties of the substrate. It is assumed that these changes do not affect the diffusion of chemicals, and specifically the diffusion coefficient $D_{v}$ does not depend on $\varepsilon$ (nonetheless, we allow for $D_{v}$ to be a smooth enough function of the spatial variable $x$ ). This is consistent with in vitro experiments where the cells are positioned on a micropatterned surface, and hence their random and chemotactic motility are affected by the microstructure, whereas the chemoatractant diffuses freely in the solution above the surface [19].

In order to specify the dependence of the model coefficients on the microscopic scale $\varepsilon$, we introduce the concept of a spatial dynamical system as follows (see, e.g., [8]). We consider a probability space $(\Omega, \mathcal{F}, P)$ with probability measure $P$. Throughout the paper, $\Omega$ is assumed to be a compact metric space and $\mathcal{F}$ is the $\sigma$-algebra of Borel sets over $\Omega$. We define a spatial dynamical system $\mathcal{T}(x): \Omega \rightarrow \Omega$, i.e. a family $\left\{\mathcal{T}(x): x \in \mathbb{R}^{d}\right\}$ of invertible maps, such that for each $x \in \mathbb{R}^{d}$, both $\mathcal{T}(x)$ and $\mathcal{T}^{-1}(x)$ are measurable and satisfy the following conditions:

(i) $\mathcal{T}(0)$ is the identity map on $\Omega$ and $\mathcal{T}(x)$ satisfies the semigroup property:

$$
\mathcal{T}\left(x_{1}+x_{2}\right)=\mathcal{T}\left(x_{1}\right) \mathcal{T}\left(x_{2}\right) \quad \text { for all } x_{1}, x_{2} \in \mathbb{R}^{d} .
$$

(ii) $P$ is an invariant measure for $\mathcal{T}(x)$, i.e. for each $x \in \mathbb{R}^{d}$ and $F \in \mathcal{F}$ we have that

$$
P\left(\mathcal{T}^{-1}(x) F\right)=P(F) .
$$


(iii) For each $F \in \mathcal{F}$, the set $\left\{(x, \omega) \in \mathbb{R}^{d} \times \Omega: \mathcal{T}(x) \omega \in F\right\}$ is a $d x \times d P(\omega)$-measurable subset of $\mathbb{R}^{d} \times \Omega$, where $d x$ denotes the Lebesgue measure on $\mathbb{R}^{d}$.

The coefficients in (1) are defined as follows. First, we define two stationary random fields through the relations

$$
D_{u}(x, \omega)=\widetilde{D}_{u}(\mathcal{T}(x) \omega) \text { and } \chi(x, \omega)=\widetilde{\chi}(\mathcal{T}(x) \omega),
$$

where $\widetilde{D}_{u}$ and $\tilde{\chi}$ are given measurable functions over $\Omega$. Then, given the specified assumptions on the random fields, the coefficients $D_{u}^{\varepsilon}(x)$ and $\chi^{\varepsilon}(x)$ are defined as

$$
D_{u}^{\varepsilon}(x)=D_{u}(x / \varepsilon, \omega) \text { and } \chi^{\varepsilon}(x)=\chi(x / \varepsilon, \omega) .
$$

From a mathematical point of view, this construction of the coefficients is common in the stochastic homogenization literature because it allows for the use of ergodic theory in the asymptotic investigation of (1) as $\varepsilon \rightarrow 0$ (see section 4). From a modeling perspective, this construction is equivalent to the assumption that the coefficients are statistically homogeneous (see, e.g., [14]). As alluded to above, the chemoattractant diffusion coefficient $D_{v}$ does not depend on $\varepsilon$.

As an example, we discuss here a specific construction of $(\Omega, \mathcal{F}, P)$ and $\mathcal{T}(x)$ based on the Poisson point process in order to provide some intuition on the abstract setting discussed above. Consider the case where motile cells are positioned on a micropatterned surface with randomly imprinted "dots," i.e. $D_{u}^{\varepsilon}(x)$ and $\chi^{\varepsilon}(x)$ are assumed to attain distinct values in the union of randomly dispersed balls and in their exterior. Then, a realization $\omega \in \Omega$ is identified with a set $\omega=\left\{B\left(\boldsymbol{\alpha}_{m}\right): m \in \mathbb{N}\right\}$ of a spatial distribution of balls $B\left(\boldsymbol{\alpha}_{m}\right)$ of a specified radius centered at $\boldsymbol{\alpha}_{m}$, and the $\sigma$-algebra $\mathcal{F}$ is defined as follows. Let $N(\omega, A)$ denote the number of balls the centers of which fall in the open set $A \subset \mathbb{R}^{2}$. Then, $\mathcal{F}$ is the $\sigma$-algebra generated by the subsets of $\Omega$ of the form

$$
\left\{\omega \in \Omega: N\left(\omega, A_{1}\right)=k_{1}, \ldots, N\left(\omega, A_{i}\right)=k_{i}\right\},
$$

where $i, k_{1}, \ldots, k_{i}$ are non-negative integers and $A_{1}, \ldots, A_{i}$ are disjoint open sets. A natural choice for the probability measure $P$ (in the absence of any a priori information) is given by the Poisson point process defined in the following way. We let

$$
P\left(N\left(\omega, A_{1}\right)=k_{1}, \ldots, N\left(\omega, A_{i}\right)=k_{i}\right)=P\left(N\left(\omega, A_{1}\right)=k_{1}\right) \times \ldots \times P\left(N\left(\omega, A_{i}\right)=k_{i}\right),
$$

with

$$
P(N(\omega, A)=k)=\frac{(\lambda|A|)^{k}}{k !} \exp (-\lambda|A|),
$$

where $\lambda$ is a positive parameter. In this setting, $\mathcal{T}(x)$ is defined as the family of translation operators given by:

$$
\mathcal{T}(x) \omega=\left\{B\left(\boldsymbol{\alpha}_{m}\right)+x: m \in \mathbb{N}\right\},
$$

where $x \in \mathbb{R}^{2}$ and $\omega=\left\{B\left(\boldsymbol{\alpha}_{m}\right): m \in \mathbb{N}\right\}$. One can define a metric that turns $\Omega$ into a compact metric space, as required in the more general setting of section 2 . This can be achieved either by considering an alternative characterization of the Poisson point process as a point process over i.i.d. compact domains that cover the Euclidean space 
(see, e.g., [14]) or by using an appropriate weighting and normalization of one of the standard sequence space norms (see, e.g., [29]). This specific construction of $(\Omega, \mathcal{F}, P)$ and $\mathcal{T}(x)$ is intuitive from a modeling perspective. Nonetheless, the somewhat more abstract setting of a spatial dynamical system is quite versatile, and will be adopted in the remainder of the paper.

The following assumption is used throughout the paper.

Assumption 1. The following hold:

(i) It is assumed that $0<d_{u}^{0} \leq \widetilde{D}_{u}(\omega) \leq d_{u}^{1}<\infty$ and $0 \leq \widetilde{\chi}(\omega) \leq \chi^{1}<\infty$ for P-a.s. $\omega \in \Omega$.

(ii) It is assumed that $D_{v} \in W^{2, \infty}(Q)$ is strongly elliptic, i.e.,

$$
\begin{array}{r}
0<d_{v}^{0} \leq\left(D_{v}(x) \xi, \xi\right) \leq d_{v}^{1}<\infty \text { for } x \in Q \text { and } \xi \in \mathbb{R}^{d}, \\
\text { and } \sup _{Q}\left|\nabla D_{v}(x)\right|+\sup _{Q}\left|\nabla^{2} D_{v}(x)\right| \leq d_{v}^{2} \text {, and } \alpha, \gamma \text { are positive constants. }
\end{array}
$$

(iii) With respect to the initial conditions, it is assumed that

$$
u_{0} \in H^{1}(Q), v_{0} \in H^{2}(Q), \text { and } u_{0}(x) \geq 0, v_{0}(x) \geq 0 \text { for a.e. } x \in Q \text {. }
$$

Moreover, if $d=\operatorname{dim}(Q)=2$ or $d=3$, it is additionally assumed that

$$
\begin{aligned}
\left(1+|Q|^{\frac{2-r}{2}}\left\|u_{0}\right\|_{L^{1}(Q)}^{r / 2}\right)\left[\max \left\{\left\|u_{0}\right\|_{L^{r}(Q)}, C_{g}\left(\left\|u_{0}\right\|_{L^{1}(Q)}+\left\|u_{0}\right\|_{L^{1}(Q)}^{\frac{2}{d+2}}\right)\right\}\right. \\
\left.+\left\|\nabla v_{0}\right\|_{L^{q}(Q)}\right]<\frac{2 d_{u}^{0}}{r} \frac{1}{\chi^{1} C_{b} C_{v}},
\end{aligned}
$$

where $q=\max \{2+\zeta, d\}, 1+\frac{\zeta}{4+\zeta}<r \leq 2$ for any $\zeta>0$ if $d=2$, and $\frac{d}{2}<r \leq 2$ if $d=3$. The constants $C_{v}, C_{b}$, and $C_{g}$ appear in the estimates (20) and (22)-(25).

We are now in a position to define the concept of weak solution that is used in this paper. In the following, $Q_{\tau}=(0, \tau) \times Q$ for $\tau>0$, and $\langle\cdot, \cdot\rangle_{Q_{\tau}}$ denotes the integral $\langle u, v\rangle_{Q_{\tau}}=\int_{0}^{\tau} \int_{Q} u v d x d t$.

Definition 2. The pair $\left(u^{\varepsilon}, v^{\varepsilon}\right)$ is a weak solution of (1) if $u^{\varepsilon} \in L^{2}\left(0, \tau ; H^{1}(Q)\right) \cap$ $H^{1}\left(0, \tau ; L^{2}(Q)\right), v^{\varepsilon} \in L^{4}\left(0, \tau ; W^{1,4}(Q)\right) \cap H^{1}\left(0, \tau ; L^{2}(Q)\right)$, and

$$
\begin{aligned}
& \left\langle u_{t}^{\varepsilon}, \phi\right\rangle_{Q_{\tau}}+\left\langle D_{u}^{\varepsilon}(x) \nabla u^{\varepsilon}-\chi^{\varepsilon}(x) u^{\varepsilon} \nabla v^{\varepsilon}, \nabla \phi\right\rangle_{Q_{\tau}}=0, \\
& \left\langle v_{t}^{\varepsilon}, \psi\right\rangle_{Q_{\tau}}+\left\langle D_{v}(x) \nabla v^{\varepsilon}, \nabla \psi\right\rangle_{Q_{\tau}}+\gamma\left\langle v^{\varepsilon}, \psi\right\rangle_{Q_{\tau}}=\alpha\left\langle u^{\varepsilon}, \psi\right\rangle_{Q_{\tau}},
\end{aligned}
$$

for any $\phi, \psi \in L^{2}\left(0, \tau ; H^{1}(Q)\right)$ and $P$-a.s. in $\Omega$. Moreover, $u^{\varepsilon}$ and $v^{\varepsilon}$ satisfy the initial conditions $u^{\varepsilon}(0, x)=u_{0}(x), v^{\varepsilon}(0, x)=v_{0}(x)$ in $L^{2}(Q)$ for $P$-a.s. $\omega \in \Omega$.

\section{Existence of solutions of the microscopic problem and a priori estimates}

In this section, we establish a priori estimates for the weak solutions of (1) that eventually lead to the proof of our main homogenization result in section 4 . In what follows, we distinguish (and treat differently) the cases $\operatorname{dim}(Q)=1$ and $\operatorname{dim}(Q) \geq 2$. In the latter case, motivated by experimental and modeling settings for biological and 
physical systems, we only consider the cases $\operatorname{dim}(Q)=2$ and $\operatorname{dim}(Q)=3$. However similar results can also be obtained when $\operatorname{dim}(Q) \geq 4$.

If $\operatorname{dim}(Q)=1$, the chemotaxis system has a global solution as shown in $[22,41$, 46]. However, since the system studied in this paper has fast oscillating diffusion and chemotaxis coefficients, we provide a different proof of the well-posedness of the system than the one developed in $[22,41,46]$. Specifically, our derivation of the a priori estimates does not require the differentiability of $D_{u}^{\varepsilon}$ or $\chi^{\varepsilon}$.

Theorem 3. Under Assumption 1 and $\operatorname{dim}(Q)=1$ there exists a unique weak solution of (1) for every $\varepsilon>0$, and for P-a.s. $\omega \in \Omega$ we have

$$
\begin{aligned}
& \left\|u^{\varepsilon}\right\|_{L^{\infty}\left(0, \tau ; L^{2}(Q)\right)}+\left\|\partial_{x} u^{\varepsilon}\right\|_{L^{\infty}\left(0, \tau ; L^{2}(Q)\right)}+\left\|\partial_{t} u^{\varepsilon}\right\|_{L^{2}\left(Q_{\tau}\right)} \leq C, \\
& \left\|v^{\varepsilon}\right\|_{L^{\infty}\left(0, \tau ; H^{1}(Q)\right)}+\left\|\partial_{t} v^{\varepsilon}\right\|_{L^{2}\left(0, \tau ; H^{1}(Q)\right)}+\left\|\partial_{x}^{2} v^{\varepsilon}\right\|_{L^{\infty}\left(0, \tau ; L^{2}(Q)\right)} \leq C,
\end{aligned}
$$

for any $\tau>0$, where the constant $C$ is independent of $\varepsilon$.

Proof. The existence of a weak solution to problem (1) is proved by showing the existence of a fix point of the operator $K$ defined on $L^{4}\left(0, \tau ; W^{1,4}(Q)\right)$ by $v^{\varepsilon}=K\left(\bar{v}^{\varepsilon}\right)$ with $v^{\varepsilon}$ given as a solution of the linear problem

$$
\begin{aligned}
u_{t}^{\varepsilon}= & \partial_{x} \cdot\left(D_{u}^{\varepsilon}(x) \partial_{x} u^{\varepsilon}-\chi^{\varepsilon}(x) u^{\varepsilon} \partial_{x} \bar{v}^{\varepsilon}\right) & & \text { in } Q_{\tau}, \\
v_{t}^{\varepsilon}= & \partial_{x} \cdot\left(D_{v}(x) \partial_{x} v^{\varepsilon}\right)-\gamma v^{\varepsilon}+\alpha u^{\varepsilon} & & \text { in } Q_{\tau}, \\
& \partial_{x} u^{\varepsilon}=0, \quad \partial_{x} v^{\varepsilon}=0 & & \text { on }(0, \tau) \times \partial Q, \\
& u^{\varepsilon}(0, x)=u_{0}(x), \quad v^{\varepsilon}(0, x)=v_{0}(x) & & \text { in } Q .
\end{aligned}
$$

By applying Galerkin's method [17] and a priori estimates similar to the estimates (10), (15), (17), and (18) established below, we obtain for every $\bar{v}^{\varepsilon} \in L^{4}\left(0, \tau ; W^{1,4}(Q)\right)$ the existence of solutions $\left(u^{\varepsilon}, v^{\varepsilon}\right)$ of $(6)$ with $u^{\varepsilon} \in L^{2}\left(0, \tau ; H^{1}(Q)\right) \cap H^{1}\left(0, \tau ; L^{2}(Q)\right)$ and $v^{\varepsilon} \in H^{1}\left(0, \tau ; L^{2}(Q)\right) \cap L^{\infty}\left(0, \tau ; H^{2}(Q)\right)$. Then, the compact embedding $L^{4}\left(0, \tau ; H^{2}(Q)\right) \cap$ $H^{1}\left(0, \tau ; L^{2}(Q)\right) \subset L^{4}\left(0, \tau ; W^{1,4}(Q)\right)$, along with the Schauder Fixed point theorem and a priori estimates ensure the existence of a solution to the original nonlinear problem (1) for all $\varepsilon>0$. The regularity of the solutions ensures that $u^{\varepsilon}, v^{\varepsilon} \in C\left([0, \tau] ; L^{2}(Q)\right)$ for $P$-a.s. $\omega \in \Omega$, and thus the initial conditions are satisfied.

We also remark that the a priori estimates are first derived for Galerkin approximations constructed by smooth eigenfunctions of the one-dimensional Laplace operator with Neumann boundary conditions. Then, using standard arguments pertaining to the weak convergence and lower semicontinuity of the norms involved, we also obtain the corresponding estimates for the solutions $u^{\varepsilon}$ and $v^{\varepsilon}$ of (1).

We remark that, provided Assumption 1, the solutions of (1) remain nonnegative for all times, see e.g. [41, 45]. To prove the required a priori estimates, we first consider $\phi=1$ and $\psi=1$ as test functions in (3) and (4) to obtain

$$
\left\|u^{\varepsilon}(t)\right\|_{L^{1}(Q)}=\left\|u_{0}\right\|_{L^{1}(Q)} \quad \text { for } t \geq 0
$$

and

$$
\partial_{t}\left\|v^{\varepsilon}(t)\right\|_{L^{1}(Q)}=-\gamma\left\|v^{\varepsilon}(t)\right\|_{L^{1}(Q)}+\alpha\left\|u^{\varepsilon}(t)\right\|_{L^{1}(Q)} \quad \text { for } t>0 .
$$

Hence, we obtain

$$
\left\|v^{\varepsilon}(t)\right\|_{L^{1}(Q)}=\left\|v_{0}\right\|_{L^{1}(Q)} e^{-\gamma t}+\alpha \gamma^{-1}\left(1-e^{-\gamma t}\right)\left\|u_{0}\right\|_{L^{1}(Q)} \quad \text { for } t \geq 0 .
$$


Multiplying the second equation in (1) by $v^{\varepsilon}$ and $\partial_{x}^{2} v^{\varepsilon}$, integrating over $Q$, and using zero-flux boundary conditions together with the specified assumptions on $D_{v}$, we have

$$
\begin{aligned}
& \frac{1}{2} \partial_{t}\left\|v^{\varepsilon}(t)\right\|_{L^{2}(Q)}^{2}+d_{v}^{0}\left\|\partial_{x} v^{\varepsilon}(t)\right\|_{L^{2}(Q)}^{2}+\gamma\left\|v^{\varepsilon}(t)\right\|_{L^{2}(Q)}^{2} \leq \alpha\left\|u^{\varepsilon}(t)\right\|_{L^{2}(Q)}\left\|v^{\varepsilon}(t)\right\|_{L^{2}(Q)} \\
& \frac{1}{2} \partial_{t}\left\|\partial_{x} v^{\varepsilon}(t)\right\|_{L^{2}(Q)}^{2}+d_{v}^{0}\left\|\partial_{x}^{2} v^{\varepsilon}(t)\right\|_{L^{2}(Q)}^{2}+\gamma\left\|\partial_{x} v^{\varepsilon}(t)\right\|_{L^{2}(Q)}^{2} \\
& \quad \leq \alpha\left\|u^{\varepsilon}(t)\right\|_{L^{2}(Q)}\left\|\partial_{x}^{2} v^{\varepsilon}(t)\right\|_{L^{2}(Q)}+d_{v}^{2}\left\|\partial_{x} v^{\varepsilon}(t)\right\|_{L^{2}(Q)}\left\|\partial_{x}^{2} v^{\varepsilon}(t)\right\|_{L^{2}(Q)}
\end{aligned}
$$

Applying Young's and Gronwall's inequalities and using $v_{0} \in H^{1}(Q)$ yield

$$
\begin{aligned}
\left\|v^{\varepsilon}\right\|_{L^{\infty}\left(0, \tau ; L^{2}(Q)\right)}+\left\|\partial_{x} v^{\varepsilon}\right\|_{L^{\infty}\left(0, \tau ; L^{2}(Q)\right)}+\left\|\partial_{x}^{2} v^{\varepsilon}\right\|_{L^{2}\left(Q_{\tau}\right)} & \leq C_{1}\left\|u^{\varepsilon}\right\|_{L^{2}\left(Q_{\tau}\right)}+C_{2},
\end{aligned}
$$

where the constants $C_{1}$ and $C_{2}$ are independent of $\varepsilon$.

Multiplying the first equation in (1) by $u^{\varepsilon}$, integrating over $Q$, and using zero-flux boundary conditions together with the stated assumptions on $\widetilde{D}_{u}$ give

$$
\partial_{t}\left\|u^{\varepsilon}(t)\right\|_{L^{2}(Q)}^{2}+2 d_{u}^{0}\left\|\partial_{x} u^{\varepsilon}(t)\right\|_{L^{2}(Q)}^{2} \leq 2\left\langle\chi^{\varepsilon}(x) u^{\varepsilon}(t) \partial_{x} v^{\varepsilon}(t), \partial_{x} u^{\varepsilon}(t)\right\rangle_{Q} .
$$

The term on the right-hand side can be estimated as

$$
\left\langle\chi^{\varepsilon}(x) u^{\varepsilon} \partial_{x} v^{\varepsilon}, \partial_{x} u^{\varepsilon}\right\rangle_{Q} \leq \frac{\left(\chi^{1}\right)^{2}}{d_{u}^{0}}\left\|u^{\varepsilon} \partial_{x} v^{\varepsilon}\right\|_{L^{2}(Q)}^{2}+\frac{d_{u}^{0}}{4}\left\|\partial_{x} u^{\varepsilon}\right\|_{L^{2}(Q)}^{2} .
$$

We use the Gagliardo-Nirenberg inequality, i.e. for $w \in W^{1, l}(Q)$ we use

$$
\|w\|_{L^{s}(Q)} \leq \tilde{C}\left(\|\nabla w\|_{L^{l}(Q)}^{\sigma}\|w\|_{L^{q}(Q)}^{1-\sigma}+\|w\|_{L^{1}(Q)}\right), \quad \frac{1}{s}=\sigma\left[\frac{1}{l}-\frac{1}{d}\right]+(1-\sigma) \frac{1}{q},
$$

with (a) $d=\operatorname{dim}(Q)=1, s=4, \sigma=1 / 2, l=2, q=1$, (b) $d=1, s=2, \sigma=1 / 3, l=2$, $q=1$, and (c) $d=1, s=4, \sigma=1 / 4, l=2, q=2$, respectively, to obtain

$$
\begin{aligned}
\left\|u^{\varepsilon}\right\|_{L^{4}(Q)} & \leq \tilde{C}\left(\left\|\partial_{x} u^{\varepsilon}\right\|_{L^{2}(Q)}^{1 / 2}\left\|u^{\varepsilon}\right\|_{L^{1}(Q)}^{1 / 2}+\left\|u^{\varepsilon}\right\|_{L^{1}(Q)}\right), \\
\left\|u^{\varepsilon}\right\|_{L^{2}(Q)} & \leq \tilde{C}\left(\left\|\partial_{x} u^{\varepsilon}\right\|_{L^{2}(Q)}^{1 / 3}\left\|u^{\varepsilon}\right\|_{L^{1}(Q)}^{2 / 3}+\left\|u^{\varepsilon}\right\|_{L^{1}(Q)}\right), \\
\left\|\partial_{x} v^{\varepsilon}\right\|_{L^{4}(Q)} & \leq \tilde{C}\left(\left\|\partial_{x}^{2} v^{\varepsilon}\right\|_{L^{2}(Q)}^{1 / 4}\left\|\partial_{x} v^{\varepsilon}\right\|_{L^{2}(Q)}^{3 / 4}+\left\|\partial_{x} v^{\varepsilon}\right\|_{L^{2}(Q)}\right) .
\end{aligned}
$$

Thus, using estimate (14) we have

$$
\begin{aligned}
\int_{0}^{\tau}\left\|\partial_{x} v^{\varepsilon}\right\|_{L^{4}(Q)}^{4} d t & \leq 8 \tilde{C} \int_{0}^{\tau}\left[\left\|\partial_{x}^{2} v^{\varepsilon}\right\|_{L^{2}(Q)}\left\|\partial_{x} v^{\varepsilon}\right\|_{L^{2}(Q)}^{3}+\left\|\partial_{x} v^{\varepsilon}\right\|_{L^{2}(Q)}^{4}\right] d t \\
& \leq C\left[\sup _{(0, \tau)}\left\|\partial_{x} v^{\varepsilon}\right\|_{L^{2}(Q)}^{3}\left\|\partial_{x}^{2} v^{\varepsilon}\right\|_{L^{2}\left(Q_{\tau}\right)}+\sup _{(0, \tau)}\left\|\partial_{x} v^{\varepsilon}\right\|_{L^{2}(Q)}^{4}\right] .
\end{aligned}
$$

Then, the estimate in (10) together with (13) ensure that

$$
\begin{aligned}
\left\|\partial_{x} v^{\varepsilon}\right\|_{L^{4}\left(Q_{\tau}\right)}^{4} & \leq C_{1}\left[\left\|u^{\varepsilon}\right\|_{L^{2}\left(Q_{\tau}\right)}^{4}+1\right] \\
& \leq C_{2}\left[\left\|\partial_{x} u^{\varepsilon}\right\|_{L^{2}\left(Q_{\tau}\right)}^{4 / 3} \sup _{(0, \tau)}\left\|u^{\varepsilon}\right\|_{L^{1}(Q)}^{8 / 3}+\sup _{(0, \tau)}\left\|u^{\varepsilon}\right\|_{L^{1}(Q)}^{4}+1\right] .
\end{aligned}
$$


Hence, using the last inequality along with (7) and (12), we obtain

$$
\begin{aligned}
& \frac{\left(\chi^{1}\right)^{2}}{d_{u}^{0}}\left\|u^{\varepsilon} \partial_{x} v^{\varepsilon}\right\|_{L^{2}\left(Q_{\tau}\right)}^{2} \leq \frac{d_{u}^{0}}{8 \tilde{C}\left(\left\|u_{0}\right\|_{L^{1}(Q)}^{2}+1\right)}\left\|u^{\varepsilon}\right\|_{L^{4}\left(Q_{\tau}\right)}^{4}+C_{1}\left\|\partial_{x} v^{\varepsilon}\right\|_{L^{4}\left(Q_{\tau}\right)}^{4} \\
& \leq \frac{d_{u}^{0}}{8}\left\|\partial_{x} u^{\varepsilon}\right\|_{L^{2}\left(Q_{\tau}\right)}^{2}+C_{2}\left\|\partial_{x} u^{\varepsilon}\right\|_{L^{2}\left(Q_{\tau}\right)}^{4 / 3}+C_{3} \leq \frac{d_{u}^{0}}{4}\left\|\partial_{x} u^{\varepsilon}\right\|_{L^{2}\left(Q_{\tau}\right)}^{2}+C_{4} .
\end{aligned}
$$

Combining all estimates together, we have that

$$
\left\|u^{\varepsilon}\right\|_{L^{\infty}\left(0, \tau ; L^{2}(Q)\right)}+\left\|\partial_{x} u^{\varepsilon}\right\|_{L^{2}\left(Q_{\tau}\right)} \leq C,
$$

where the constant $C$ is independent of $\varepsilon$. Using $\operatorname{dim}(Q)=1$ in the last estimate, we obtain that

$$
\left\|u^{\varepsilon}\right\|_{L^{2}\left(0, \tau ; L^{\infty}(Q)\right)} \leq C .
$$

Considering $\partial_{t} \partial_{x}^{2} v^{\varepsilon}$ as a test function in (4), applying integration by parts, and using zero-flux boundary conditions together with the specified assumptions on $D_{v}$ yield that

$$
\begin{array}{r}
\int_{0}^{\tau}\left[\left\|\partial_{t} \partial_{x} v^{\varepsilon}\right\|_{L^{2}(Q)}^{2}+\frac{d_{v}^{0}}{2} \partial_{t}\left\|\partial_{x}^{2} v^{\varepsilon}\right\|_{L^{2}(Q)}^{2}\right. \\
\left.+\frac{\gamma}{2} \partial_{t}\left\|\partial_{x} v\right\|_{L^{2}(Q)}^{2}\right] d t \leq \alpha \int_{0}^{\tau}\left|\left\langle\partial_{x} u^{\varepsilon}, \partial_{t} \partial_{x} v^{\varepsilon}\right\rangle_{Q}\right| d t \\
+\int_{0}^{\tau}\left|\left\langle\partial_{x}^{2} D_{v}(x) \partial_{x} v^{\varepsilon}+\partial_{x} D_{v}(x) \partial_{x}^{2} v^{\varepsilon}, \partial_{t} \partial_{x} v^{\varepsilon}\right\rangle_{Q}\right| d t \\
\leq \frac{1}{4} \int_{0}^{\tau}\left\|\partial_{t} \partial_{x} v^{\varepsilon}\right\|_{L^{2}(Q)}^{2} d t+C \int_{0}^{\tau}\left[\left\|\partial_{x} u^{\varepsilon}\right\|_{L^{2}(Q)}^{2}+\left\|\partial_{x}^{2} v^{\varepsilon}\right\|_{L^{2}(Q)}^{2}+\left\|\partial_{x} v^{\varepsilon}\right\|_{L^{2}(Q)}^{2}\right] d t
\end{array}
$$

where $C=C\left(d_{v}^{2}, \alpha\right)$. Then using (10), (15), and the assumption $v_{0} \in H^{2}(Q)$, we have

$$
\left\|\partial_{t} \partial_{x} v^{\varepsilon}\right\|_{L^{2}\left(Q_{\tau}\right)}+\left\|\partial_{x}^{2} v^{\varepsilon}\right\|_{L^{\infty}\left(0, \tau ; L^{2}(Q)\right)}+\left\|\partial_{x} v\right\|_{L^{\infty}\left(0, \tau ; L^{2}(Q)\right)} \leq C .
$$

Multiplying the first equation in (1) by $u_{t}^{\varepsilon}$, integrating over $Q$ and using zero-flux boundary conditions we obtain

$$
\left\|\partial_{t} u^{\varepsilon}(t)\right\|_{L^{2}(Q)}^{2}+\left\langle D_{u}^{\varepsilon}(x) \partial_{x} u^{\varepsilon}(t), \partial_{t} \partial_{x} u^{\varepsilon}(t)\right\rangle_{Q}=\left\langle\chi^{\varepsilon}(x) u^{\varepsilon}(t) \partial_{x} v^{\varepsilon}(t), \partial_{t} \partial_{x} u^{\varepsilon}(t)\right\rangle_{Q} .
$$

Then, the term on the right-hand side can be rewritten as

$$
\begin{array}{r}
\left\langle\chi^{\varepsilon}(x) u^{\varepsilon} \partial_{x} v^{\varepsilon}, \partial_{t} \partial_{x} u^{\varepsilon}\right\rangle_{Q}=\left\langle\chi^{\varepsilon}(x) \partial_{t} u^{\varepsilon} \partial_{x} v^{\varepsilon}+\chi^{\varepsilon}(x) u^{\varepsilon} \partial_{t} \partial_{x} v^{\varepsilon}, \partial_{x} u^{\varepsilon}\right\rangle_{Q} \\
+\partial_{t}\left\langle\chi^{\varepsilon}(x) u^{\varepsilon} \partial_{x} v^{\varepsilon}, \partial_{x} u^{\varepsilon}\right\rangle_{Q} .
\end{array}
$$

The first and second terms can be estimated as

$$
\left|\left\langle\chi^{\varepsilon}(x) \partial_{t} u^{\varepsilon} \partial_{x} v^{\varepsilon}, \partial_{x} u^{\varepsilon}\right\rangle_{Q}\right| \leq \frac{1}{2}\left\|\partial_{t} u^{\varepsilon}\right\|_{L^{2}(Q)}^{2}+\frac{\left(\chi^{1}\right)^{2}}{2}\left\|\partial_{x} v^{\varepsilon}\right\|_{L^{\infty}(Q)}^{2}\left\|\partial_{x} u^{\varepsilon}\right\|_{L^{2}(Q)}^{2},
$$

and

$$
\left|\left\langle\chi^{\varepsilon}(x) u^{\varepsilon} \partial_{t} \partial_{x} v^{\varepsilon}, \partial_{x} u^{\varepsilon}\right\rangle_{Q}\right| \leq\left(\chi^{1}\right)^{2}\left\|\partial_{t} \partial_{x} v^{\varepsilon}\right\|_{L^{2}(Q)}^{2}+\frac{1}{4}\left\|u^{\varepsilon}\right\|_{L^{\infty}(Q)}^{2}\left\|\partial_{x} u^{\varepsilon}\right\|_{L^{2}(Q)}^{2} .
$$


Thus, considering the fact that $\left\|\partial_{x} v^{\varepsilon}\right\|_{L^{\infty}\left(Q_{\tau}\right)} \leq C$, we obtain

$$
\begin{aligned}
\left\|\partial_{t} u^{\varepsilon}\right\|_{L^{2}(Q)}^{2} & +d_{u}^{0} \partial_{t}\left\|\partial_{x} u^{\varepsilon}\right\|_{L^{2}(Q)}^{2} \leq C_{1}\left(\left\|\partial_{x} u^{\varepsilon}\right\|_{L^{2}(Q)}^{2}+\left\|\partial_{t} \partial_{x} v^{\varepsilon}\right\|_{L^{2}(Q)}^{2}\right) \\
& +C_{2}\left\|u^{\varepsilon}\right\|_{L^{\infty}(Q)}^{2}\left\|\partial_{x} u^{\varepsilon}\right\|_{L^{2}(Q)}^{2}+2 \partial_{t}\left\langle\chi^{\varepsilon}(x) u^{\varepsilon} \partial_{x} v^{\varepsilon}, \partial_{x} u^{\varepsilon}\right\rangle_{Q} .
\end{aligned}
$$

For the last term we have that for $t \in(0, \tau]$

$$
\int_{0}^{t} \partial_{s}\left\langle\chi^{\varepsilon} u^{\varepsilon} \partial_{x} v^{\varepsilon}, \partial_{x} u^{\varepsilon}\right\rangle_{Q} d s=\left\langle\chi^{\varepsilon} u^{\varepsilon}(t) \partial_{x} v^{\varepsilon}(t), \partial_{x} u^{\varepsilon}(t)\right\rangle_{Q}-\left\langle\chi^{\varepsilon} u^{\varepsilon}(0) \partial_{x} v^{\varepsilon}(0), \partial_{x} u^{\varepsilon}(0)\right\rangle_{Q}
$$

and

$$
\begin{aligned}
& \left|\left\langle\chi^{\varepsilon} u^{\varepsilon}(t) \partial_{x} v^{\varepsilon}(t), \partial_{x} u^{\varepsilon}(t)\right\rangle_{Q}\right|+\left|\left\langle\chi^{\varepsilon} u^{\varepsilon}(0) \partial_{x} v^{\varepsilon}(0), \partial_{x} u^{\varepsilon}(0)\right\rangle_{Q}\right| \leq \frac{d_{u}^{0}}{8}\left\|\partial_{x} u^{\varepsilon}(t)\right\|_{L^{2}(Q)}^{2} \\
& \quad+C_{1}\left\|\partial_{x} v^{\varepsilon}(t)\right\|_{L^{\infty}(Q)}^{2}\left\|u^{\varepsilon}(t)\right\|_{L^{2}(Q)}^{2}+C_{2}\left\|\partial_{x} v_{0}\right\|_{L^{\infty}(Q)}^{2}\left\|u_{0}\right\|_{L^{2}(Q)}^{2}+C_{3}\left\|\partial_{x} u_{0}\right\|_{L^{2}(Q)}^{2}
\end{aligned}
$$

Applying Gronwall's lemma and using estimates (15), (16), and (17) along with $u_{0} \in$ $H^{1}(Q)$ and $v_{0} \in H^{2}(Q)$, we obtain that for a.e. $t \in[0, \tau]$

$$
\left\|\partial_{x} u^{\varepsilon}(t)\right\|_{L^{2}(Q)}^{2} \leq C_{1} \exp \left(\left\|u^{\varepsilon}\right\|_{L^{2}\left(0, \tau ; L^{\infty}(Q)\right)}^{2}\right)+C_{2} \leq C .
$$

Thus, we conclude that

$$
\left\|\partial_{x} u^{\varepsilon}\right\|_{L^{\infty}\left(0, \tau ; L^{2}(Q)\right)}^{2}+\left\|\partial_{t} u^{\varepsilon}\right\|_{L^{2}\left(Q_{\tau}\right)}^{2} \leq C .
$$

To prove uniqueness, we assume there are two solutions and consider $u^{\varepsilon}=u_{1}^{\varepsilon}-u_{2}^{\varepsilon}$ and $v^{\varepsilon}=v_{1}^{\varepsilon}-v_{2}^{\varepsilon}$ as test functions in equations (3) and (4), respectively,

$$
\begin{aligned}
& \left\langle u_{t}^{\varepsilon}, u^{\varepsilon}\right\rangle_{Q_{\tau}}+\left\langle D_{u}^{\varepsilon}(x) \partial_{x} u^{\varepsilon}, \partial_{x} u^{\varepsilon}\right\rangle_{Q_{\tau}}-\left\langle\chi^{\varepsilon}(x)\left(u^{\varepsilon} \partial_{x} v_{1}^{\varepsilon}+u_{2}^{\varepsilon} \partial_{x} v^{\varepsilon}\right), \partial_{x} u^{\varepsilon}\right\rangle_{Q_{\tau}}=0, \\
& \left\langle v_{t}^{\varepsilon}, v^{\varepsilon}\right\rangle_{Q_{\tau}}+\left\langle D_{v}(x) \partial_{x} v^{\varepsilon}, \partial_{x} v^{\varepsilon}\right\rangle_{Q_{\tau}}+\gamma\left\langle v^{\varepsilon}, v^{\varepsilon}\right\rangle_{Q_{\tau}}=\alpha\left\langle u^{\varepsilon}, v^{\varepsilon}\right\rangle_{Q_{\tau}}
\end{aligned}
$$

Then using the boundedness of $u_{i}^{\varepsilon}$ and $\partial_{x} v_{i}^{\varepsilon}, i=1,2$, along with Young's and Gronwall's inequalities, we obtain $u_{1}^{\varepsilon}=u_{2}^{\varepsilon}$ and $v_{1}^{\varepsilon}=v_{2}^{\varepsilon}$ for a.e. $(t, x) \in Q_{\tau}$ and $P$-a.s. $\omega \in \Omega$.

Remark. The constant $C$ in estimates (5) depends on $\tau$, i.e. $C \sim a e^{b \tau}, a, b>0$. However, if $\operatorname{dim}(Q)=1$ the solutions of (1) exist for any fixed $\tau>0$ without any smallness restrictions on $u_{0}$ and $v_{0}$. Moreover, the estimates (5) are uniform in $\varepsilon$.

In the system investigated in this paper, the diffusion $D_{u}^{\varepsilon}$ and chemotaxis $\chi^{\varepsilon}$ coefficients depend on a small parameter $\varepsilon$, and we do not have uniform in $\varepsilon$ estimates for $\nabla D_{u}^{\varepsilon}$ and $\nabla \chi^{\varepsilon}$. Hence, when $\operatorname{dim}(Q)=2$ we cannot use the derivation of the a priori estimates and the corresponding proof of well-posedness developed in [39]. Instead, when $\operatorname{dim}(Q)=2$ or $\operatorname{dim}(Q)=3$ we adopt an approach similar to the one in [11].

Theorem 4. Under Assumption 1 and assuming $d=\operatorname{dim}(Q)=2$ or 3 , there exists a unique weak solution of (1) for every $\varepsilon>0$, and we have

$$
\begin{aligned}
\left\|u^{\varepsilon}\right\|_{L^{\infty}\left(0, \tau ; L^{2}(Q)\right)}+\left\|\nabla u^{\varepsilon}\right\|_{L^{\infty}\left(0, \tau ; L^{2}(Q)\right)}+\left\|\partial_{t} u^{\varepsilon}\right\|_{L^{2}\left(Q_{\tau}\right)} & \leq C, \\
\left\|v^{\varepsilon}\right\|_{L^{\infty}\left(0, \tau ; H^{1}(Q)\right)}+\left\|\partial_{t} v^{\varepsilon}\right\|_{L^{2}\left(0, \tau ; H^{1}(Q)\right)}+\left\|v^{\varepsilon}\right\|_{L^{2}\left(0, \tau ; H^{2}(Q)\right)} & \leq C
\end{aligned}
$$

for $P$-a.s. $\omega \in \Omega$ and a constant $C$ which is independent of $\varepsilon$. 
Proof. Similarly to Theorem 3 we obtain the non-negativity and the estimates (7) and (8) for the $L^{1}$-norms of $u^{\varepsilon}$ and $v^{\varepsilon}$.

Using the estimates for the derivatives of the Green function of the operator $A=$ $-\nabla \cdot\left(D_{v}(x) \nabla\right)$ (see, e.g., $\left.[11,36,48]\right)$ we obtain

$$
\left\|\nabla e^{-t(A+\gamma)} \phi\right\|_{L^{r_{1}}(Q)} \leq C_{1} t^{-\frac{1}{2}-\frac{d}{2}\left(\frac{1}{r_{2}}-\frac{1}{r_{1}}\right)}\|\phi\|_{L^{r_{2}(Q)}}, \quad t>0,
$$

for all $1 \leq r_{2} \leq r_{1} \leq \infty$ and $\phi \in L^{r_{2}}(Q)$, and

$$
\left\|\nabla e^{-t(A+\gamma)} \phi\right\|_{L^{p}(Q)} \leq C_{2}\|\nabla \phi\|_{L^{p}(Q)},
$$

for $2 \leq p \leq \infty, \phi \in W^{1, p}(Q)$, and some constants $C_{1}$ and $C_{2}$ that depend on $Q$. Here, $\gamma$ is the decay constant in the second equation in (1). Applying the variation-of-constants formula, see e.g. [44], yields

$$
v^{\varepsilon}(t, \cdot)=e^{-t(A+\gamma)} v_{0}(\cdot)+\alpha \int_{0}^{t} e^{(s-t)(A+\gamma)} u^{\varepsilon}(s, \cdot) d s .
$$

Then, for $r_{1}$ and $r_{2}$ such that $\frac{1}{2}+\frac{d}{2}\left(\frac{1}{r_{2}}-\frac{1}{r_{1}}\right)<1$, we have

$$
\left\|\nabla v^{\varepsilon}(\cdot, t)\right\|_{L^{r_{1}(\Omega)}} \leq C_{v}\left(\left\|\nabla v_{0}\right\|_{L^{r_{1}(Q)}}+\sup _{s \in(0, t)}\left\|u^{\varepsilon}(\cdot, s)\right\|_{L^{r_{2}(Q)}}\right) \quad \text { for all } t \in(0, \tau]
$$

We now consider $\left|u^{\varepsilon}\right|^{p-1}$, for some $p>1$, as a test function in (3) to obtain

$$
\frac{d}{d t} \int_{Q}\left|u^{\varepsilon}\right|^{p} d x+\left.\left.4 \frac{p-1}{p} d_{u}^{0} \int_{Q}|\nabla| u^{\varepsilon}\right|^{\frac{p}{2}}\right|^{2} d x \leq\left. 2(p-1) \chi^{1} \int_{Q}\left|u^{\varepsilon}\right|^{\frac{p}{2}}|\nabla| u^{\varepsilon}\right|^{\frac{p}{2}}|| \nabla v^{\varepsilon} \mid d x .
$$

The integral on the right-hand side can be rewritten as

$$
I=\left.\int_{Q}\left|u^{\varepsilon}\right|^{\frac{p}{2}}|\nabla| u^{\varepsilon}\right|^{\frac{p}{2}}|| \nabla v^{\varepsilon}\left|d x \leq\left\|\nabla\left|u^{\varepsilon}\right|^{\frac{p}{2}}\right\|_{L^{2}(Q)}\left\|\left|u^{\varepsilon}\right|^{\frac{p}{2}}\right\|_{L^{q_{1}}(Q)}\left\|\nabla v^{\varepsilon}\right\|_{L^{q_{2}(Q)}},\right.
$$

where $1 / q_{1}+1 / q_{2}=1 / 2$.

For $d=2$ and any $\zeta>0$, we consider $q_{2}=2+\zeta$ and $q_{1}=2+\frac{4}{\zeta}$. Then, applying the Sobolev embedding and estimate (20) with $1+\frac{\zeta}{4+\zeta}<r_{2} \leq 2$, we obtain

$$
\begin{aligned}
I & \leq\left\|\nabla\left|u^{\varepsilon}\right|^{\frac{p}{2}}\right\|_{L^{2}(Q)}\left\|\left|u^{\varepsilon}\right|^{\frac{p}{2}}\right\|_{L^{2+\frac{4}{\varsigma}}(Q)}\left\|\nabla v^{\varepsilon}\right\|_{L^{2+\zeta}(Q)} \leq\left\|\nabla\left|u^{\varepsilon}\right|^{\frac{p}{2}}\right\|_{L^{2}(Q)} \times \\
& \times C_{b}\left(\left\|\nabla\left|u^{\varepsilon}\right|^{\frac{p}{2}}\right\|_{L^{2}(Q)}+\left\|\left|u^{\varepsilon}\right|^{\frac{p}{2}}\right\|_{L^{1}(Q)}\right) C_{v}\left(\left\|\nabla v_{0}\right\|_{L^{2+\zeta}(Q)}+\sup _{s \in(0, t)}\left\|u^{\varepsilon}(s)\right\|_{\left.L^{r_{2}(Q)}()^{2}\right)}\right)
\end{aligned}
$$

where $C_{b}$ is the embedding constant. If $\left\|\nabla\left|u^{\varepsilon}(t)\right|^{\frac{p}{2}}\right\|_{L^{2}(Q)}^{2} \geq 1$ for $t \in(0, \tau]$ and $p=r_{2}$, using the estimate for $I$ and inequality (21) we obtain

$$
\begin{aligned}
& \frac{d}{d t} \int_{Q}\left|u^{\varepsilon}\right|^{r_{2}} d x \leq 2\left(r_{2}-1\right)\left\|\nabla\left|u^{\varepsilon}\right|^{\frac{r_{2}}{2}}\right\|_{L^{2}(Q)}^{2}\left[C_{b} C_{v} \chi^{1}\left(1+\left\|u_{0}\right\|_{L^{1}(Q)}^{r_{2} / 2}|Q|^{\frac{2-r_{2}}{2}}\right) \times\right. \\
&\left.\times\left(\left\|\nabla v_{0}\right\|_{L^{2+\zeta}(Q)}+\sup _{s \in(0, t)}\left\|u^{\varepsilon}(s)\right\|_{L^{r_{2}(Q)}}\right)-\frac{2 d_{u}^{0}}{r_{2}}\right] .
\end{aligned}
$$


If for some $t \in(0, \tau]$ we have that $\left\|\nabla\left|u^{\varepsilon}(t)\right|^{\frac{r_{2}}{2}}\right\|_{L^{2}(Q)} \leq 1$, then using the GagliardoNirenberg inequality (11) with $s=2, \sigma=1 / 2, d=2, l=2$, and $q=1$ we obtain that

$$
\begin{array}{r}
\left\|u^{\varepsilon}(t)\right\|_{L^{r_{2}}(Q)}^{r_{2}} \leq \tilde{C}\left(\left\|\nabla\left|u^{\varepsilon}(t)\right|^{\frac{r_{2}}{2}}\right\|_{L^{2}(Q)}\left\|\left|u^{\varepsilon}\right|^{\frac{r_{2}}{2}}\right\|_{L^{1}(Q)}+\left\|\left|u^{\varepsilon}\right|^{\frac{r_{2}}{2}}\right\|_{L^{1}(Q)}^{2}\right) \\
\leq C_{g}\left(\left\|u_{0}\right\|_{L^{1}(Q)}^{\frac{r_{2}}{2}}+\left\|u_{0}\right\|_{L^{1}(Q)}^{r_{2}}\right) .
\end{array}
$$

For $d=3$ we consider $q_{2}=3, q_{1}=6$, and we apply the Sobolev embedding theorem to obtain

$$
\begin{array}{r}
I \leq\left\|\nabla\left|u^{\varepsilon}\right|^{\frac{p}{2}}\right\|_{L^{2}(Q)} C_{b}\left(\left\|\nabla\left|u^{\varepsilon}\right|^{\frac{p}{2}}\right\|_{L^{2}(Q)}+\left\|\left|u^{\varepsilon}\right|^{\frac{p}{2}}\right\|_{L^{1}(Q)}\right) \times \\
\times C_{v}\left(\left\|\nabla v_{0}\right\|_{L^{3}(Q)}+\sup _{s \in(0, t)}\left\|u^{\varepsilon}(s)\right\|_{L^{r_{2}(Q)}}\right),
\end{array}
$$

where $C_{b}$ is the embedding constant and $3 / 2<r_{2} \leq 2$. If $\left\|\nabla\left|u^{\varepsilon}(t)\right|^{\frac{p}{2}}\right\|_{L^{2}(Q)} \geq 1$ for $t \in(0, \tau]$ and $p=r_{2}$, we have

$$
\begin{aligned}
& \frac{d}{d t} \int_{Q}\left|u^{\varepsilon}\right|^{r_{2}} d x \leq 2\left(r_{2}-1\right)\left\|\nabla\left|u^{\varepsilon}\right|^{\frac{r_{2}}{2}}\right\|_{L^{2}(Q)}^{2} \times \\
& \times\left[C_{b} C_{v} \chi^{1}\left(1+\left\|u_{0}\right\|_{L^{1}(Q)}^{r_{2} / 2}|Q|^{\frac{2-r_{2}}{2}}\right)\left(\left\|\nabla v_{0}\right\|_{L^{3}(Q)}+\sup _{s \in(0, t)}\left\|u^{\varepsilon}(s)\right\|_{L^{r_{2}}(Q)}\right)-\frac{2 d_{u}^{0}}{r_{2}}\right] .
\end{aligned}
$$

If for some $t \in(0, \tau]$ we have $\left\|\nabla\left|u^{\varepsilon}(t)\right|^{\frac{r_{2}}{2}}\right\|_{L^{2}(Q)} \leq 1$, then using the Gagliardo-Nirenberg inequality (11) with $s=2, \sigma=3 / 5, d=3, l=2$, and $q=1$ we obtain that

$$
\begin{array}{r}
\left\|u^{\varepsilon}(t)\right\|_{L^{r_{2}}(Q)}^{r_{2}} \leq \tilde{C}\left(\left\|\nabla\left|u^{\varepsilon}(t)\right|^{\frac{r_{2}}{2}}\right\|_{L^{2}(Q)}^{\frac{6}{5}}\left\|\left|u^{\varepsilon}\right|^{\frac{r_{2}}{2}}\right\|_{L^{1}(Q)}^{\frac{4}{5}}+\left\|\left|u^{\varepsilon}\right|^{\frac{r_{2}}{2}}\right\|_{L^{1}(Q)}^{2}\right) \\
\leq C_{g}\left(\left\|u_{0}\right\|_{L^{1}(Q)}^{\frac{2 r_{2}}{5}}+\left\|u_{0}\right\|_{L^{1}(Q)}^{r_{2}}\right) .
\end{array}
$$

Thus, if $\left(1+\left\|u_{0}\right\|_{L^{1}(Q)}^{\frac{r_{2}}{2}}|Q|^{\frac{2-r_{2}}{2}}\right)\left(\left\|\nabla v_{0}\right\|_{L^{q}(Q)}+\sup _{s \in(0, t)}\left\|u^{\varepsilon}(\cdot, s)\right\|_{L^{r_{2}(Q)}}\right)$ is sufficiently small we obtain that $\left\|u^{\varepsilon}(t)\right\|_{L^{r_{2}(Q)}}^{r_{2}}$ is monotone decreasing for all $t \in(0, \tau]$ such that $\left\|\nabla\left|u^{\varepsilon}(t)\right|^{\frac{r_{2}}{2}}\right\|_{L^{2}(Q)} \geq 1$. Here, $q=\max \{2+\zeta, d\}$ for any $\zeta>0$. For any $t \in(0, \tau]$ such that $\left\|\nabla\left|u^{\varepsilon}(t)\right|^{\frac{r_{2}}{2}}\right\|_{L^{2}(Q)} \leq 1$ we have

$$
\left\|u^{\varepsilon}(t)\right\|_{L^{r_{2}}(Q)} \leq C_{g}\left(\left\|u_{0}\right\|_{L^{1}(Q)}^{\frac{2}{d^{+2}}}+\left\|u_{0}\right\|_{L^{1}(Q)}\right) .
$$

Hence, if $v_{0}$ and $u_{0}$ satisfy assumption (2), then

$$
\left\|u^{\varepsilon}\right\|_{L^{\infty}\left(0, \tau ; L^{r_{2}}(Q)\right)} \leq \max \left\{\left\|u_{0}\right\|_{L^{r_{2}}(Q)}, C_{g}\left(\left\|u_{0}\right\|_{L^{1}(Q)}+\left\|u_{0}\right\|_{L^{1}(Q)}^{\frac{2}{d+2}}\right)\right\} .
$$

Using the last estimate and taking $u^{\varepsilon}$ as a test function in (3) we have

$$
\left\|u^{\varepsilon}\right\|_{L^{\infty}\left(0, \tau ; L^{2}(Q)\right)}+\left\|\nabla u^{\varepsilon}\right\|_{L^{2}\left(Q_{\tau}\right)} \leq C,
$$

with a constant $C$ independent of $\varepsilon$. Considering $v^{\varepsilon}$ and $\partial_{t} v^{\varepsilon}$ as test functions in (4) we obtain

$\left\|v^{\varepsilon}\right\|_{L^{\infty}\left(0, \tau ; L^{2}(Q)\right)}+\left\|\partial_{t} v^{\varepsilon}\right\|_{L^{2}\left(Q_{\tau}\right)}+\left\|\nabla v^{\varepsilon}\right\|_{L^{\infty}\left(0, \tau ; L^{2}(Q)\right)} \leq C_{1}\left(\left\|u^{\varepsilon}\right\|_{L^{2}\left(Q_{\tau}\right)}+\left\|v_{0}\right\|_{H^{1}(Q)}\right) \leq C$. 
Taking $\left|u^{\varepsilon}\right|^{p-1}$ as a test function in (3) with $p>d$ yields

$$
\left\|u^{\varepsilon}\right\|_{L^{\infty}\left(0, \tau ; L^{p}(Q)\right)} \leq C .
$$

Thus, applying (20) and using the estimate (27) with $p>d$, we obtain

$$
\left\|\nabla v^{\varepsilon}\right\|_{L^{\infty}\left(Q_{\tau}\right)} \leq C_{1}\left\|u^{\varepsilon}\right\|_{L^{\infty}\left(0, \tau ; L^{p}(Q)\right)} \leq C_{2} .
$$

Then, considering $\partial_{t} u^{\varepsilon}$ as a test function in (3) ensure

$$
\left\|\partial_{t} u^{\varepsilon}\right\|_{L^{2}\left(Q_{\tau}\right)}+\left\|\nabla u^{\varepsilon}\right\|_{L^{\infty}\left(0, \tau, L^{2}(Q)\right)} \leq C .
$$

Taking $\Delta v^{\varepsilon}$ and $\Delta \partial_{t} v^{\varepsilon}$ as test functions in (4) and applying zero Neumann boundary conditions for $u^{\varepsilon}$ result in

$$
\left\|\partial_{t} \nabla v^{\varepsilon}\right\|_{L^{2}\left(Q_{\tau}\right)}+\left\|\nabla^{2} v^{\varepsilon}\right\|_{L^{\infty}\left(0, \tau ; L^{2}(Q)\right)} \leq C .
$$

As in Theorem 3 we obtain the existence of a weak solution of (1) in $Q_{\tau}$ by applying the Galerkin method and a fixed point argument. Similarly, we show the uniqueness of the weak solution of (1) by considering the equations for the difference of two solutions and showing that they are equal a.e. in $Q_{\tau}$ and $P$-a.s. in $\Omega$.

\section{Stochastic homogenization}

In this section, we derive our main homogenization result for problem (1). The system of macroscopic equations is obtained in Theorem 15 by using the concept of stochastic two-scale convergence introduced in [52]. For the reader's convenience we state the general definition of two-scale convergence by means of Palm measures, and then apply it to the specific context of the problem studied in this paper. In the following, we also make use of the notions of invariance and ergodicity, which we now define.

Definition 5. A measurable function $f$ on $\Omega$ is said to be invariant for a dynamical system $\mathcal{T}(x)$ if for each $x \in \mathbb{R}^{d}, f(\omega)=f(\mathcal{T}(x) \omega), P$-a.s. on $\Omega$.

Definition 6. A dynamical system $\mathcal{T}(x)$ is said to be ergodic, if every measurable function which is invariant for $\mathcal{T}(x)$ is $P$-a.s. equal to a constant.

The random environment described by the coefficients in (1) can also be characterized in terms of a random measure, which is defined as follows.

Definition 7. Let $(\Omega, \mathcal{F})$ be a measurable space and $\mathcal{B}\left(\mathbb{R}^{d}\right)$ be the $\sigma$-algebra of Borel sets in $\mathbb{R}^{d}$. A mapping $\tilde{\mu}: \Omega \times \mathcal{B}\left(\mathbb{R}^{d}\right) \rightarrow \mathbb{R}_{+} \cup\{\infty\}$ is called a random measure on $\left(\mathbb{R}^{d}, \mathcal{B}\left(\mathbb{R}^{d}\right)\right)$ if the function $\mu_{\omega}(A)=\tilde{\mu}(\omega, A)$ is $\mathcal{F}$-measurable in $\omega \in \Omega$ for each $A \in \mathcal{B}\left(\mathbb{R}^{d}\right)$ and a measure in $A \in \mathcal{B}\left(\mathbb{R}^{d}\right)$ for each $\omega \in \Omega$.

Even though more general definitions of a random measure exist in the literature (see, e.g., [14] or [28]), in the remainder of the paper $\mu_{\omega}$ will always denote a random measure on $\left(\mathbb{R}^{d}, \mathcal{B}\left(\mathbb{R}^{d}\right)\right)$. 
Definition 8. The Palm measure of the random measure $\mu_{\omega}$ is the measure $\boldsymbol{\mu}$ on $(\Omega, \mathcal{F})$ defined by the relation

$$
\boldsymbol{\mu}(A)=\int_{\Omega} \int_{\mathbb{R}^{d}} \mathbb{I}_{[0,1)^{d}}(x) \mathbb{I}_{A}(\mathcal{T}(x) \omega) d \mu_{\omega}(x) d P(\omega),
$$

where $\mathbb{I}_{K}$ denotes the characteristic function of the set $K$.

The value of the notion of a Palm measure is that it allows for a generalization of Birkhoff's ergodic theorem for stationary random measures. Specifically, given a dynamical system $\mathcal{T}(x)$, we say that the random measure $\mu_{\omega}$ is stationary if for every $\phi \in C_{0}^{\infty}\left(\mathbb{R}^{d}\right)$

$$
\int_{\mathbb{R}^{d}} \phi(y-x) d \mu_{\omega}(y)=\int_{\mathbb{R}^{d}} \phi(y) d \mu_{\mathcal{T}(x) \omega}(y) .
$$

The intensity $m\left(\mu_{\omega}\right)$ of a random measure $\mu_{\omega}$ is defined by

$$
m\left(\mu_{\omega}\right)=\int_{\Omega} \int_{[0,1)^{d}} d \mu_{\omega}(x) d P(\omega) .
$$

Theorem 9 (Ergodic theorem [52]). Let the dynamical system $\mathcal{T}(x)$ be ergodic and assume that the stationary random measure $\mu_{\omega}$ has finite intensity $m\left(\mu_{\omega}\right)>0$. Then

$$
\lim _{t \rightarrow \infty} \frac{1}{t|A|} \int_{t A} g(\mathcal{T}(x) \omega) d \mu_{\omega}(x)=\int_{\Omega} g(\omega) d \boldsymbol{\mu}(\omega) \quad \text { a.s. with respect to } P
$$

for all bounded Borel sets $A$, with volume $|A|>0$, and all $g \in L^{1}(\Omega, \boldsymbol{\mu})$.

We remark that for $\boldsymbol{\mu}=P$ (i.e., $d \mu_{\omega}(x)=d x$ ), Theorem 9 reduces to the classical ergodic theorem of Birkhoff.

We now define the notion of stochastic two-scale convergence, which is one of the main tools used in proving Theorem 15. We consider the family of random measures

$$
d \mu_{\omega}^{\varepsilon}(x)=\varepsilon^{d} d \mu_{\omega}\left(\frac{x}{\varepsilon}\right) .
$$

We remark that an immediate consequence of Theorem 9 is that on every compact subset of $\mathbb{R}^{d}$, the family $d \mu_{\omega}^{\varepsilon}(x)$ converges weakly to the deterministic measure $m\left(\mu_{\omega}\right) d x$ a.s. with respect to $P$ as $\varepsilon \rightarrow 0$ (see, e.g., [52]).

Definition 10 (Stochastic two-scale convergence [21, 52]). Let $Q$ be a domain in $\mathbb{R}^{d}$, $\mathcal{T}(x)$ be an ergodic dynamical system, and $\mathcal{T}(x) \tilde{\omega}$ be a "typical trajectory," i.e. one that satisfies equation (30) for all $g \in C(\Omega)$. Then, we say that a sequence $\left\{v^{\varepsilon}\right\} \subset$ $L^{2}\left(0, \tau ; L^{2}\left(Q, \mu_{\tilde{\omega}}^{\varepsilon}\right)\right)$ converges stochastically two-scale to $v \in L^{2}\left(0, \tau ; L^{2}(Q \times \Omega, d x \times\right.$ $d \boldsymbol{\mu}(\omega)))$ if

$$
\limsup _{\varepsilon \rightarrow 0} \int_{0}^{\tau} \int_{Q}\left|v^{\varepsilon}(t, x)\right|^{2} d \mu_{\tilde{\omega}}^{\varepsilon}(x) d t<\infty
$$

and

$$
\begin{aligned}
\lim _{\varepsilon \rightarrow 0} \int_{0}^{\tau} \int_{Q} v^{\varepsilon}(t, x) \varphi(t, x) b & (\mathcal{T}(x / \varepsilon) \tilde{\omega}) d \mu_{\tilde{\omega}}^{\varepsilon}(x) d t \\
& =\int_{0}^{\tau} \int_{Q} \int_{\Omega} v(t, x, \omega) \varphi(t, x) b(\omega) d \boldsymbol{\mu}(\omega) d x d t
\end{aligned}
$$

for all $\varphi \in C_{0}^{\infty}([0, \tau) \times Q)$ and $b \in L^{2}(\Omega, \boldsymbol{\mu})$. 
It is evident that if $Q \subset \mathbb{R}^{d}$ is bounded, each $\varphi \in C^{\infty}\left(\bar{Q}_{\tau}\right)$ can be used as a test function in Definition 10. The concept of a "typical trajectory" in Definition 10 extends to realizations $\tilde{\omega} \in \Omega$. Specifically, we say that $\tilde{\omega} \in \Omega$ is a "typical realization" if (30) holds true at $\tilde{\omega}$ for all $g \in C(\Omega)$.

Theorem 11. [21, 52] Every sequence $\left\{v^{\varepsilon}\right\} \subset L^{2}\left(0, \tau ; L^{2}\left(Q, \mu_{\tilde{\omega}}^{\varepsilon}\right)\right)$ that satisfies (31) converges along a subsequence to some $v \in L^{2}\left(0, \tau ; L^{2}(Q \times \Omega, d x \times d \boldsymbol{\mu}(\omega))\right)$ in the sense of stochastic two-scale convergence.

Before we proceed, we need to define a concept of stochastic derivative and the space $H^{1}(\Omega, \boldsymbol{\mu})$ for the Palm measure $\boldsymbol{\mu}$. First, we say that a function $u \in C(\Omega)$ belongs to $C^{1}(\Omega)$ if the limit

$$
\partial_{\omega}^{j} u(\omega)=\lim _{h \rightarrow 0} \frac{u\left(\mathcal{T}\left(h e_{j}\right) \omega\right)-u(\omega)}{h}
$$

exists and $\partial_{\omega}^{j} u(\omega) \in C(\Omega)$. Then, the Sobolev space $H^{1}(\Omega, \boldsymbol{\mu})$ is defined as follows.

Definition 12. [52] We say that a function $u \in L^{2}(\Omega, \boldsymbol{\mu})$ belongs to $H^{1}(\Omega, \boldsymbol{\mu})$ and $\partial_{\omega} u$ is a (stochastic) derivative of $u$ if there exists a sequence $u_{k} \in C^{1}(\Omega)$ such that $u_{k} \rightarrow u$ in $L^{2}(\Omega, \boldsymbol{\mu})$ and $\partial_{\omega}^{j} u_{k} \rightarrow \partial_{\omega}^{j} u$ in $L^{2}(\Omega, \boldsymbol{\mu})$.

In general, the stochastic derivative $\partial_{\omega} u$ does not have to be unique (see [52] for counterexamples). We remark, however, that the particular setting of our problem yields the uniqueness of $\partial_{\omega} u$. We also define $L_{\mathrm{pot}}^{2}(\Omega, \boldsymbol{\mu})$ and $L_{\mathrm{sol}}^{2}(\Omega, \boldsymbol{\mu})$ to be the spaces of potential functions and divergence-free functions, respectively. More precisely,

$$
L_{\mathrm{pot}}^{2}(\Omega, \boldsymbol{\mu})=\overline{\left\{\partial_{\omega} u: u \in C^{1}(\Omega)\right\}} \text { and } L_{\mathrm{sol}}^{2}(\Omega, \boldsymbol{\mu})=\left(L_{\mathrm{pot}}^{2}(\Omega, \boldsymbol{\mu})\right)^{\perp},
$$

where the closure in the definition of $L_{\mathrm{pot}}^{2}(\Omega, \boldsymbol{\mu})$ is with respect to the $L^{2}(\Omega, \boldsymbol{\mu})$ norm.

We now state two compactness results for the notion of stochastic two-scale convergence to be used in the following. Theorems 13 and 14 were proved in [52] in the more general setting of an arbitrary random measure. Here, the theorems are stated in the context of our problem, i.e. for a non-degenerate random measure $\mu_{\omega}$ (see [52] for the definition of non-degeneracy).

Remark. For a non-degenerate measure, $\partial_{\omega}^{j}$ denotes the generator of a strongly continuous group of unitary operators in $L^{2}(\Omega, \boldsymbol{\mu})$ associated with $\mathcal{T}(x)$ along the $e_{j}$ direction. The domains of $\partial_{\omega}^{j}$, with $j=1, \ldots, d$, are dense in $L^{2}(\Omega, \boldsymbol{\mu})$. We let $\nabla_{\omega} u=$ $\left(\partial_{\omega}^{1} u, \ldots, \partial_{\omega}^{d} u\right)^{T}$ and $H^{1}(\Omega, \boldsymbol{\mu})=\left\{v \in L^{2}(\Omega, \boldsymbol{\mu}): \nabla_{\omega} v \in L^{2}(\Omega, \boldsymbol{\mu})\right\}$.

Theorem 13. [52] Let $Q$ be a domain in $\mathbb{R}^{d}$ and assume that $\mu_{\omega}$ is a non-degenerate random measure and that the sequence $\left\{v^{\varepsilon}\right\} \subset H^{1}\left(Q, \mu_{\tilde{\omega}}^{\varepsilon}\right)$ is such that

$$
\left\|v^{\varepsilon}\right\|_{L^{2}\left(Q, \mu_{\tilde{\omega}}^{\varepsilon}\right)} \leq C(\tilde{\omega}), \quad\left\|\nabla v^{\varepsilon}\right\|_{L^{2}\left(Q, \mu_{\tilde{\omega}}^{\varepsilon}\right)} \leq C(\tilde{\omega}) .
$$

Then there exist functions $v \in H^{1}(Q)$ and $v_{1} \in L^{2}\left(Q ; L_{\text {pot }}^{2}(\Omega, \boldsymbol{\mu})\right)$ such that, up to a subsequence, the following hold:

$$
\begin{array}{cl}
v^{\varepsilon}-v & \text { stochastically two-scale, } \\
\nabla v^{\varepsilon} \rightarrow \nabla_{x} v+v_{1} & \text { stochastically two-scale. }
\end{array}
$$


Theorem 14. [52] Let $Q$ be a domain in $\mathbb{R}^{d}$ and assume that $\mu_{\omega}$ is a non-degenerate random measure and that the sequence $\left\{v^{\varepsilon}\right\} \subset H^{1}\left(Q, \mu_{\tilde{\omega}}^{\varepsilon}\right)$ is such that

$$
\left\|v^{\varepsilon}\right\|_{L^{2}\left(Q, \mu_{\tilde{\omega}}^{\varepsilon}\right)} \leq C(\tilde{\omega}), \quad \varepsilon\left\|\nabla v^{\varepsilon}\right\|_{L^{2}\left(Q, \mu_{\tilde{\omega}}^{\varepsilon}\right)} \leq C(\tilde{\omega}) .
$$

Then there exists a function $v \in L^{2}\left(Q ; H^{1}(\Omega, \boldsymbol{\mu})\right)$ such that, up to a subsequence, the following hold:

$$
\begin{array}{cl}
v^{\varepsilon} \rightarrow v & \text { stochastically two-scale, } \\
\varepsilon \nabla v^{\varepsilon} \rightarrow \nabla_{\omega} v & \text { stochastically two-scale. }
\end{array}
$$

Similar results hold for $\left\{v^{\varepsilon}\right\} \subset L^{2}\left(0, \tau ; H^{1}\left(Q, \mu_{\tilde{\omega}}^{\varepsilon}\right)\right)$, where the time variable is considered as a parameter [21].

In the following theorems, the Palm measure reduces to the probability measure $P$, i.e., $\boldsymbol{\mu}=P$. We now state and prove the main homogenization result of this paper.

Theorem 15. We assume that the dynamical system $\mathcal{T}(x)$ is ergodic and that the coefficients $D_{u}^{\varepsilon}, \chi^{\varepsilon}$, and $D_{v}$ along with the initial conditions $u_{0}$ and $v_{0}$ satisfy Assumption 1 . Then, the sequence of weak solutions $\left\{u^{\varepsilon}, v^{\varepsilon}\right\}$ of the microscopic problem (1) converges strongly in $L^{2}\left(Q_{\tau}\right)$ and weakly in $L^{2}\left(0, \tau ; H^{1}(Q)\right)$ to the solution $(u, v) \in L^{2}\left(0, \tau ; H^{1}(Q)\right)^{2}$ of the macroscopic model:

$$
\begin{array}{ll}
\partial_{t} u=\nabla \cdot\left(D^{*} \nabla u-\chi^{*} u \nabla v\right) & \text { in } Q_{\tau}, \\
\partial_{t} v=\nabla \cdot\left(D_{v}(x) \nabla v\right)-\gamma v+\alpha u & \text { in } Q_{\tau}, \\
\left(D^{*} \nabla u-\chi^{*} u \nabla v\right) \cdot n=0, \quad \nabla v \cdot n=0 & \text { on }(0, \tau) \times \partial Q, \\
u(0, x)=u_{0}(x), \quad v(0, x)=v_{0}(x) & \text { in } Q,
\end{array}
$$

$P$-a.s. in $\Omega$. The effective (macroscopic) diffusion and chemotaxis matrices are defined as

$$
D^{*} \xi=\int_{\Omega} \widetilde{D}_{u}(\omega)\left(\bar{u}_{1, \xi}+\xi\right) d P(\omega), \quad \chi^{*} \xi=-\int_{\Omega}\left(\widetilde{D}_{u}(\omega) \hat{u}_{1, \xi}-\widetilde{\chi}(\omega) \xi\right) d P(\omega)
$$

for any $\xi \in \mathbb{R}^{d}$, where $\bar{u}_{1, \xi}, \hat{u}_{1, \xi}$ are solutions of the auxiliary problems

$$
\begin{array}{lll}
\bar{u}_{1, \xi} \in L_{\text {pot }}^{2}(\Omega) & \text { such that } & \widetilde{D}_{u}(\omega)\left(\bar{u}_{1, \xi}+\xi\right) \in L_{\text {sol }}^{2}(\Omega), \\
\hat{u}_{1, \xi} \in L_{p o t}^{2}(\Omega) & \text { such that } & \widetilde{D}_{u}(\omega) \hat{u}_{1, \xi}-\widetilde{\chi}(\omega) \xi \in L_{\text {sol }}^{2}(\Omega) .
\end{array}
$$

Proof. From the a priori estimates in (5), we obtain that

$$
u^{\varepsilon}, \nabla u^{\varepsilon}, \partial_{t} u^{\varepsilon}, v^{\varepsilon}, \nabla v^{\varepsilon}, \nabla^{2} v^{\varepsilon}, \partial_{t} v^{\varepsilon}, \partial_{t} \nabla v^{\varepsilon}
$$

are bounded sequences in $L^{2}\left(Q_{\tau}\right)$ for $P$-a.s. $\omega \in \Omega$. Then, using Theorem 13 with $\boldsymbol{\mu}=P$, we obtain that, up to a subsequence,

$$
\begin{array}{cl}
u^{\varepsilon} \rightarrow u & \text { stochastically two-scale, } \quad u \in L^{2}\left(0, \tau ; H^{1}(Q)\right), \\
\nabla u^{\varepsilon} \rightarrow \nabla u+u_{1} & \text { stochastically two-scale, } \quad u_{1} \in L^{2}\left(Q_{\tau} ; L_{\text {pot }}^{2}(\Omega)\right), \\
\partial_{t} u^{\varepsilon} \rightarrow \tilde{u} & \text { stochastically two-scale, } \quad \tilde{u} \in L^{2}\left(Q_{\tau} ; L^{2}(\Omega)\right), \\
v^{\varepsilon} \rightarrow v & \text { stochastically two-scale, } \quad v \in L^{2}\left(0, \tau ; H^{1}(Q)\right), \\
\partial_{t} v^{\varepsilon} \rightarrow \tilde{v} & \text { stochastically two-scale, } \quad \tilde{v} \in L^{2}\left(0, \tau ; H^{1}(Q)\right), \\
\nabla v^{\varepsilon} \rightarrow \hat{v} & \text { stochastically two-scale, } \quad \hat{v} \in L^{2}\left(0, \tau ; H^{1}(Q)\right)
\end{array}
$$


for all "typical" realizations $\omega$.

Now, considering the stochastic two-scale convergence of $u^{\varepsilon}$ and $\partial_{t} u^{\varepsilon}$, we have that for $\varphi \in C_{0}^{\infty}\left(Q_{\tau}\right), b \in L^{2}(\Omega)$ and any "typical" realization $\tilde{\omega} \in \Omega$

$$
\begin{aligned}
& \int_{Q_{\tau}} \int_{\Omega} \tilde{u}(t, x, \omega) \varphi(t, x) b(\omega) d P(\omega) d x d t=\lim _{\varepsilon \rightarrow 0} \int_{Q_{\tau}} \partial_{t} u^{\varepsilon}(t, x) \varphi(t, x) b(\mathcal{T}(x / \varepsilon) \tilde{\omega}) d x d t= \\
& -\lim _{\varepsilon \rightarrow 0} \int_{Q_{\tau}} u^{\varepsilon}(t, x) \partial_{t} \varphi(t, x) b(\mathcal{T}(x / \varepsilon) \tilde{\omega}) d x d t=-\int_{Q_{\tau}} \int_{\Omega} u(t, x) \partial_{t} \varphi(t, x) b(\omega) d P(\omega) d x d t \\
& =\int_{Q_{\tau}} \int_{\Omega} \partial_{t} u(t, x) \varphi(t, x) b(\omega) d P(\omega) d x d t .
\end{aligned}
$$

Thus, $\tilde{u}(t, x, \omega)=\partial_{t} u(t, x)$ for a.e. $(t, x) \in Q_{\tau}$ and $P$-a.s. $\omega \in \Omega$. Similarly we conclude that $\tilde{v}(t, x)=\partial_{t} v(t, x)$ for a.e. $(t, x) \in Q_{\tau}$.

From the definition of stochastic two-scale convergence of $\nabla v^{\varepsilon}$, we obtain that for $\varphi \in C_{0}^{\infty}\left(Q_{\tau}\right), b \in L^{2}(\Omega)$ and any "typical" realization $\tilde{\omega} \in \Omega$

$$
\lim _{\varepsilon \rightarrow 0} \int_{Q_{\tau}} \nabla v^{\varepsilon}(t, x) \varphi(t, x) b(\mathcal{T}(x / \varepsilon) \tilde{\omega}) d x d t=\int_{Q_{\tau}} \int_{\Omega} \hat{v}(t, x) \varphi(t, x) b(\omega) d P(\omega) d x d t .
$$

The weak convergence of $v^{\varepsilon}$ in $L^{2}\left(0, \tau ; H^{1}(Q)\right)$, which is ensured by the a priori estimates, implies that

$$
\lim _{\varepsilon \rightarrow 0} \int_{Q_{\tau}} \nabla v^{\varepsilon}(t, x) \varphi(t, x) d x d t=\int_{Q_{\tau}} \nabla v(t, x) \varphi(t, x) d x d t
$$

for $P$-a.s. $\omega \in \Omega$ and $\varphi \in L^{2}\left(Q_{\tau}\right)$. Thus, by choosing $b(\omega)=1$, we conclude that $\hat{v}(t, x)=\nabla v(t, x)$ for a.e. $(t, x) \in Q_{\tau}$. Hence, the stated a priori estimates and the Aubin-Lions compactness lemma [33] ensure that, up to a subsequence, $u^{\varepsilon} \rightarrow u, v^{\varepsilon} \rightarrow v$, and $\nabla v^{\varepsilon} \rightarrow \nabla v$ strongly in $L^{2}\left(Q_{\tau}\right)$ as $\varepsilon \rightarrow 0, P$-a.s.

We now derive the macroscopic equations. Choosing $\psi \in C^{\infty}\left(\bar{Q}_{\tau}\right)$ as test function in (4), and by considering the weak convergence of $u^{\varepsilon}$ and $v^{\varepsilon}$, we obtain

$$
\left\langle v_{t}, \psi\right\rangle_{Q_{\tau}}+\left\langle D_{v}(x) \nabla v, \nabla \psi\right\rangle_{Q_{\tau}}+\gamma\langle v, \psi\rangle_{Q_{\tau}}=\alpha\langle u, \psi\rangle_{Q_{\tau}} .
$$

Now, we consider $\phi(t, x)=\varphi(t, x)+\varepsilon \varphi_{1}(t, x) \varphi_{2}(\mathcal{T}(x / \varepsilon) \omega)$, where $\varphi \in C^{\infty}\left(\bar{Q}_{\tau}\right), \quad \varphi_{1} \in$ $C_{0}^{\infty}\left(Q_{\tau}\right)$ and $\varphi_{2} \in C^{1}(\Omega)$, as test function in (3) and obtain

$$
\begin{array}{r}
\left\langle D_{u}^{\varepsilon} \nabla u^{\varepsilon}-\chi^{\varepsilon} u^{\varepsilon} \nabla v^{\varepsilon}, \nabla \varphi+\varepsilon \nabla \varphi_{1} \varphi_{2}(\mathcal{T}(x / \varepsilon) \omega)+\varphi_{1} \nabla_{\omega} \varphi_{2}(\mathcal{T}(x / \varepsilon) \omega)\right\rangle_{Q_{\tau}} \\
+\left\langle u_{t}^{\varepsilon}, \varphi+\varepsilon \varphi_{1} \varphi_{2}(\mathcal{T}(x / \varepsilon) \omega)\right\rangle_{Q_{\tau}}=0 .
\end{array}
$$

The stochastic two-scale limit in (39) and the strong convergence of $u^{\varepsilon}$ yield as $\varepsilon \rightarrow 0$

$$
\left\langle u_{t}, \varphi\right\rangle_{Q_{\tau}}+\left\langle\widetilde{D}_{u}(\omega)\left(\nabla u+u_{1}\right)-\tilde{\chi}(\omega) u \nabla v, \nabla \varphi+\varphi_{1} \nabla_{\omega} \varphi_{2}(\omega)\right\rangle_{Q_{\tau}, \Omega}=0 .
$$

Choosing $\varphi(t, x)=0$ for $(t, x) \in Q_{\tau}$ we obtain

$$
\left\langle\widetilde{D}_{u}(\omega)\left(\nabla u+u_{1}\right)-\widetilde{\chi}(\omega) u \nabla v, \varphi_{1}(t, x) \nabla_{\omega} \varphi_{2}(\omega)\right\rangle_{Q_{\tau}, \Omega}=0
$$


for every $\varphi_{1} \in C_{0}^{\infty}\left(Q_{\tau}\right)$ and $\varphi_{2} \in C^{1}(\Omega)$. Thus, we have that for $d t \times d x$-a.e. in $Q_{\tau}$

$$
\left\langle\widetilde{D}_{u}(\omega)\left(\nabla u+u_{1}\right)-\widetilde{\chi}(\omega) u \nabla v, \nabla_{\omega} \varphi_{2}\right\rangle_{\Omega}=0 .
$$

Due to the stated assumptions on $\widetilde{D}_{u}$ and $\widetilde{\chi}$ there exists a unique solution $u_{1}(t, x, \cdot) \in$ $L_{\mathrm{pot}}^{2}(\Omega)$ of (41) that depends linearly on $\nabla_{x} u(t, x)$ and $u(t, x) \nabla_{x} v(t, x)$ for a.e. $(t, x) \in$ $Q_{\tau}$, see e.g. [51]. We consider

$$
u_{1}(t, x, \omega)=\sum_{j=1}^{d} \partial_{x_{j}} u(t, x) \bar{u}_{1}^{j}(\omega)+u(t, x) \sum_{j=1}^{d} \partial_{x_{j}} v(t, x) \hat{u}_{1}^{j}(\omega)
$$

for a.e. $(t, x) \in Q_{\tau}$ and $P$-a.s. $\omega \in \Omega$, and obtain from (41) that $\bar{u}_{1}^{j}, \hat{u}_{1}^{j} \in L_{\text {pot }}^{2}(\Omega)$, for $j=1, \ldots, d$, are solutions of the problems (37) and (38), respectively. Considering now $\varphi_{1}=0$ in (40), and using the above expression for $u_{1}$, we obtain the macroscopic model (35) with effective coefficients $D^{*}$ and $\chi^{*}$ given by (36).

By the stochastic two-scale convergence of $u^{\varepsilon}$ and $\partial_{t} u^{\varepsilon}$, and the initial condition $u^{\varepsilon}(0, x)=u_{0}(x)$, we obtain for all $\varphi \in C_{0}^{\infty}([0, \tau) \times Q), b \in L^{2}(\Omega)$ and any "typical" realization $\tilde{\omega} \in \Omega$ that

$$
\begin{aligned}
& \int_{Q_{\tau}} \int_{\Omega} \partial_{t} u(t, x) \varphi(t, x) b(\omega) d P(\omega) d x d t=\lim _{\varepsilon \rightarrow 0} \int_{Q_{\tau}} \partial_{t} u^{\varepsilon}(t, x) \varphi(t, x) b(\mathcal{T}(x / \varepsilon) \tilde{\omega}) d x d t \\
& =-\lim _{\varepsilon \rightarrow 0} \int_{Q_{\tau}} u^{\varepsilon}(t, x) \partial_{t} \varphi(t, x) b(\mathcal{T}(x / \varepsilon) \tilde{\omega}) d x d t+\lim _{\varepsilon \rightarrow 0} \int_{Q} u_{0}(x) \varphi(0, x) b(\mathcal{T}(x / \varepsilon) \tilde{\omega}) d x d t \\
& =-\int_{Q_{\tau}} \int_{\Omega} u(t, x) \partial_{t} \varphi(t, x) b(\omega) d P(\omega) d x d t+\int_{Q} \int_{\Omega} u_{0}(x) \varphi(0, x) b(\omega) d P(\omega) d x d t .
\end{aligned}
$$

Similar calculations for $v^{\varepsilon}$ ensure that the initial conditions $u(0, x)=u_{0}(x)$ and $v(0, x)=$ $v_{0}(x)$ are satisfied a.e. in $Q$.

The proof of the uniqueness of the solution is similar to the corresponding proof for the microscopic problem, and hence the convergence of the whole sequences $\left\{u^{\varepsilon}\right\}$ and $\left\{v^{\varepsilon}\right\}$ follows. Since (35) has a unique solution, and $D^{*}$ and $\chi^{*}$ do not depend on $\omega$, it follows that the solution of (35) does not depend on $\omega$ either.

\section{Periodic approximation of the effective coefficients}

We now turn our attention to the problem of approximating the homogenized coefficients shown in (36) by means of a periodization procedure. The significance of such approximations is discussed in [5] and [42]. Here, we build upon the methods developed in [5] and consider the following periodization procedure.

We let $S_{\rho}=[0, \rho]^{d}$ for some $\rho>0$, and for each $\omega \in \Omega$ we consider the periodic functions

$$
D_{u, \text { per }}^{\rho}(z, \omega)=\widetilde{D}_{u}\left(\mathcal{T}\left(z\left(\bmod S_{\rho}\right)\right) \omega\right), \quad \chi_{\text {per }}^{\rho}(z, \omega)=\widetilde{\chi}\left(\mathcal{T}\left(z\left(\bmod S_{\rho}\right)\right) \omega\right) .
$$

Then for $P$-a.s. $\omega \in \Omega$, we consider the equations

$$
\begin{array}{cl}
\bar{u}_{t}^{\varepsilon}=\nabla \cdot\left(D_{u, \operatorname{per}}^{\rho}(x / \varepsilon, \omega) \nabla \bar{u}^{\varepsilon}-\chi_{\mathrm{per}}^{\rho}(x / \varepsilon, \omega) \bar{u}^{\varepsilon} \nabla \bar{v}^{\varepsilon}\right) & \text { in } Q_{\tau}, \\
\bar{v}_{t}^{\varepsilon}=\nabla \cdot\left(D_{v}(x) \nabla \bar{v}^{\varepsilon}\right)-\gamma \bar{v}^{\varepsilon}+\alpha \bar{u}^{\varepsilon} & \text { in } Q_{\tau}, \\
\nabla \bar{u}^{\varepsilon} \cdot n=0, \quad \nabla \bar{v}^{\varepsilon} \cdot n=0 & \text { on }(0, \tau)
\end{array}
$$


The equation for $\bar{u}^{\varepsilon}$ has periodic coefficients, and hence we can employ methods pertaining to periodic homogenization to obtain the effective coefficients for the corresponding macroscopic problem. However, since $D_{u, \text { per }}^{\rho}(z, \omega)$ and $\chi_{\text {per }}^{\rho}(z, \omega)$ are not ergodic anymore, the effective coefficients are not deterministic (i.e., they depend on $\omega \in \Omega$ ).

The unit cell problems that are obtained from the periodic homogenization approach are: Find $\bar{\eta}_{j}^{\rho}, \hat{\eta}_{j}^{\rho} \in H_{\text {per }}^{1}\left(S_{\rho}\right)$, for $j=1, \ldots, d$, such that

$$
\begin{aligned}
\nabla_{z} \cdot\left(D_{u, \text { per }}^{\rho}(z, \omega)\left(\nabla_{z} \bar{\eta}_{j}^{\rho}+e_{j}\right)\right)=0 & \text { in } S_{\rho}, \\
\nabla_{z} \cdot\left(D_{u, \text { per }}^{\rho}(z, \omega) \nabla_{z} \hat{\eta}_{j}^{\rho}-\chi_{\text {per }}^{\rho}(z, \omega) e_{j}\right)=0 & \text { in } S_{\rho} .
\end{aligned}
$$

Given the corrector functions $\bar{\eta}^{\rho}, \hat{\eta}^{\rho} \in H_{\text {per }}^{1}\left(S_{\rho}\right)$, the effective coefficients are then defined by

$$
\begin{aligned}
D_{\omega, i j}^{\rho} & =\frac{1}{\rho^{d}} \int_{S_{\rho}}\left(\left(D_{u, \text { per }}^{\rho}(z, \omega) \nabla_{z} \bar{\eta}_{i}^{\rho}\right)_{j}+D_{u, \text { per }}^{\rho}(z, \omega) \delta_{i j}\right) d z \\
\chi_{\omega, i j}^{\rho} & =-\frac{1}{\rho^{d}} \int_{S_{\rho}}\left(\left(D_{u, \text { per }}^{\rho}(z, \omega) \nabla_{z} \hat{\eta}_{i}^{\rho}\right)_{j}-\chi_{\text {per }}^{\rho}(z, \omega) \delta_{i j}\right) d z
\end{aligned}
$$

for $i, j=1, \ldots, d$, and the macroscopic equations read

$$
\begin{array}{ll}
\partial_{t} u^{\rho}=\nabla \cdot\left(D_{\omega}^{\rho} \nabla u^{\rho}-\chi_{\omega}^{\rho} u^{\rho} \nabla v^{\rho}\right) & \text { in } Q_{\tau}, \\
\partial_{t} v^{\rho}=\nabla \cdot\left(D_{v}(x) \nabla v^{\rho}\right)-\gamma v^{\rho}+\alpha u^{\rho} & \text { in } Q_{\tau}, \\
\left(D_{\omega}^{\rho} \nabla u^{\rho}-\chi_{\omega}^{\rho} u^{\rho} \nabla v^{\rho}\right) \cdot n=0, \quad \nabla v^{\rho} \cdot n=0 & \text { on }(0, \tau) \times \partial Q
\end{array}
$$

for $P$-a.s. $\omega \in \Omega$.

The following theorem is the key result of this section. It guarantees the convergence of the effective coefficients obtained by periodic approximation to the original effective coefficients obtained from the stochastic homogenization in the previous section.

Theorem 16. Let $D_{\omega}^{\rho}$ and $\chi_{\omega}^{\rho}$ be the effective coefficients obtained in (43) and (44), respectively. Then for $D^{*}$ and $\chi^{*}$ as in $(36)$, the following hold true

$$
\lim _{\rho \rightarrow \infty} D_{\omega, i j}^{\rho}=D_{i j}^{*} \quad P \text {-a.s., } \quad \lim _{\rho \rightarrow \infty} \chi_{\omega, i j}^{\rho}=\chi_{i j}^{*} \quad P \text {-a.s. }, \quad i, j=1, \ldots, d .
$$

Proof. First, we consider in $S_{1}=[0,1]$ the auxiliary problems

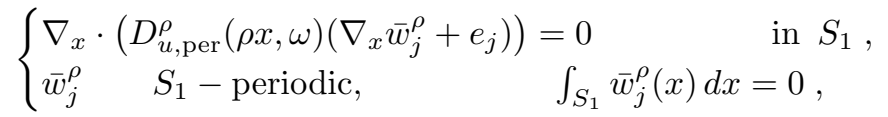

$$
\begin{aligned}
& \left\{\begin{array}{cc}
\nabla_{x} \cdot\left(D_{u, \text { per }}^{\rho}(\rho x, \omega) \nabla_{x} \hat{w}_{j}^{\rho}-\chi_{\text {per }}^{\rho}(\rho x, \omega) e_{j}\right)=0 \quad \text { in } S_{1}, \\
\hat{w}_{j}^{\rho} \quad S_{1}-\text { periodic, } & \int_{S_{1}} \hat{w}_{j}^{\rho}(x) d x=0 .
\end{array}\right.
\end{aligned}
$$

From the definition of $D_{u \text {,per }}^{\rho}$ we have that $D_{u, \text { per }}^{\rho}(\rho x, \omega)=D_{u}(\rho x, \omega)$ in $S_{1}$. Then, for $\rho=1 / \varepsilon$ and $Q=S_{1}$, one can apply the stochastic homogenization results of Section 4 to problems (46) and (47) to obtain the effective macroscopic equations

$$
\begin{array}{ccc}
\nabla_{x} \cdot\left(D^{*}\left(\nabla_{x} \bar{w}_{j}+e_{j}\right)\right)=0 & \text { in } S_{1}, & \bar{w}_{j} \quad S_{1}-\text { periodic, } \int_{S_{1}} \bar{w}_{j}(x) d x=0, \\
\nabla_{x} \cdot\left(D^{*} \nabla_{x} \hat{w}_{j}-\chi^{*} e_{j}\right)=0 & \text { in } S_{1}, & \hat{w}_{j} \quad S_{1}-\text { periodic, } \int_{S_{1}} \hat{w}_{j}(x) d x=0, \\
18
\end{array}
$$


where $j=1, \ldots, d$, and $D^{*}$ and $\chi^{*}$ are given by (36).

We then consider the coordinate transformation $y=z / \rho$ in equations (42), transforming $S_{\rho}$ to the unit interval $S_{1}$. We let $\bar{\eta}_{0, j}^{\rho}(y)=\frac{1}{\rho} \bar{\eta}_{j}^{\rho}(\rho y)$ and $\hat{\eta}_{0, j}^{\rho}(y)=\frac{1}{\rho} \hat{\eta}_{j}^{\rho}(\rho y)$, and rewrite the equations in (42) as

$$
\begin{array}{ll}
\nabla_{y} \cdot\left(D_{u, \text { per }}^{\rho}(\rho y, \omega)\left(\nabla_{y} \bar{\eta}_{0, j}^{\rho}+e_{j}\right)\right)=0 & \text { in } S_{1}, \\
\nabla_{y} \cdot\left(D_{u, \text { per }}^{\rho}(\rho y, \omega) \nabla_{y} \hat{\eta}_{0, j}^{\rho}-\chi_{\text {per }}^{\rho}(\rho y, \omega) e_{j}\right)=0 & \text { in } S_{1},
\end{array}
$$

where $\bar{\eta}_{0, j}^{\rho}$ and $\hat{\eta}_{0, j}^{\rho}$ are $S_{1}$-periodic function, $j=1, \ldots, d$. The solutions of (49) and (50) are unique up to an additive constant, which we fix by considering $\int_{S_{1}} \bar{\eta}_{0, j}^{\rho}(y) d y=0$ and $\int_{S_{1}} \hat{\eta}_{0, j}^{\rho}(y) d y=0$. Taking $\bar{\eta}_{0}^{\rho}$ and $\hat{\eta}_{0}^{\rho}$ as test functions in (49) and (50), respectively, using Assumption 1 on the coefficients $\widetilde{D}_{u}$ and $\widetilde{\chi}$, and applying the Poincaré inequality we obtain the following a priori estimates uniformly in $\rho$

$$
\left\|\bar{\eta}_{0, j}^{\rho}\right\|_{H^{1}\left(S_{1}\right)} \leq C, \quad\left\|\hat{\eta}_{0, j}^{\rho}\right\|_{H^{1}\left(S_{1}\right)} \leq C, \quad j=1, \ldots, d .
$$

Thus, we have that $\bar{\eta}_{0, j}^{\rho}$ and $\hat{\eta}_{0, j}^{\rho}$ converge weakly in $H_{\text {per }}^{1}\left(S_{1}\right)$ to $\bar{\eta}_{j}^{\infty}$ and $\hat{\eta}_{j}^{\infty}$, respectively, as $\rho \rightarrow \infty$, with $j=1, \ldots, d$. We also have that $\bar{\eta}_{0, j}^{\rho}$ and $\hat{\eta}_{0, j}^{\rho}$ converge stochastically two-scale to the same limit functions $\bar{\eta}_{j}^{\infty}=\bar{\eta}_{j}^{\infty}(y)$ and $\hat{\eta}_{j}^{\infty}=\hat{\eta}_{j}^{\infty}(y)$, with $j=1, \ldots, d$. Then, considering the results on the stochastic homogenization of equations (46) and (47), we obtain that $\bar{\eta}_{j}^{\infty}$ and $\hat{\eta}_{j}^{\infty}$ satisfy

$$
\begin{array}{ccc}
\nabla_{y} \cdot\left(D^{*}\left(\nabla_{y} \bar{\eta}_{j}^{\infty}+e_{j}\right)\right)=0 & \text { in } S_{1}, \quad \bar{\eta}_{j}^{\infty} S_{1} \text { - periodic, } \int_{S_{1}} \bar{\eta}_{j}^{\infty}(y) d y=0 \\
\nabla_{y} \cdot\left(D^{*} \nabla_{y} \hat{\eta}_{j}^{\infty}-\chi^{*} e_{j}\right)=0 & \text { in } S_{1}, \quad \hat{\eta}_{j}^{\infty} S_{1} \text { - periodic, } \int_{S_{1}} \hat{\eta}_{j}^{\infty}(y) d y=0 .
\end{array}
$$

Hence, we have that

$$
\begin{array}{rrr}
D_{u, \text { per }}^{\rho}(\rho y, \omega)\left(\nabla_{y} \bar{\eta}_{0, j}^{\rho}+e_{j}\right) & \rightarrow D^{*}\left(\nabla_{y} \bar{\eta}_{j}^{\infty}+e_{j}\right) & \text { weakly in } L^{2}\left(S_{1}\right), \\
D_{u, \text { per }}^{\rho}(\rho y, \omega) \nabla_{y} \hat{\eta}_{0, j}^{\rho}-\chi_{\text {per }}^{\rho}(\rho y, \omega) e_{j} & \rightarrow D^{*} \nabla_{y} \hat{\eta}_{j}^{\infty}-\chi^{*} e_{j} & \text { weakly in } L^{2}\left(S_{1}\right),
\end{array}
$$

as $\rho \rightarrow \infty$, for $P$-a.s. $\omega \in \Omega$ and $j=1, \ldots, d$. Finally, since the only periodic solutions of (52) with zero average are $\bar{\eta}_{j}^{\infty}(y)=0$ and $\hat{\eta}_{j}^{\infty}(y)=0$ for $y \in S_{1}$, it follows from (53) that

$$
\begin{array}{ll}
D_{\omega, j}^{\rho}=\int_{S_{1}} D_{u, \text { per }}^{\rho}(\rho y, \omega)\left(\nabla_{y} \bar{\eta}_{0, j}^{\rho}+e_{j}\right) d y & \rightarrow \int_{S_{1}} D^{*} e_{j} d y=D_{j}^{*}, \\
\chi_{\omega, j}^{\rho}=-\int_{S_{1}}\left(D_{u, \text { per }}^{\rho}(\rho y, \omega) \nabla_{y} \hat{\eta}_{0, j}^{\rho}-\chi_{\text {per }}^{\rho}(\rho y, \omega) e_{j}\right) d y & \rightarrow \int_{S_{1}} \chi^{*} e_{j} d y=\chi_{j}^{*},
\end{array}
$$

as $\rho \rightarrow \infty$, for $P$-a.s. $\omega \in \Omega$ and $j=1, \ldots, d$. This proves the convergence results stated in the theorem.

\section{Acknowledgments}

The authors would like to thank Prof. Andrey Piatnitski for advice and encouragement. AM would like to thank the Computational Science and Engineering Laboratory 
at ETH Zürich for the warm hospitality during a sabbatical semester. The research of AM is supported in part by the National Science Foundation under Grants NSF CDS\&EMSS 1521266 and NSF CAREER 1552903. The research of MP is supported in part by the EPSRC First Grant EP/K036521/1.

[1] Allaire G. (1992) Homogenization and two-scale convergence. SIAM J Math. Anal., 23, 1482-1518.

[2] Armstrong S.N., Souganidis P.E. (2012) Stochastic homogenization of Hamilton-Jacobi and degenerate Bellman equations in unbounded environments. J. Math.Pures Appl. 97, 460-504.

[3] Armstrong S.N., Souganidis P.E. (2013) Stochastic homogenization of level-set convex HamiltonJacobi equations. Int. Math. Res. Not. 17, 3420-3449.

[4] Armstrong S.N., Tran H.V., and Yu Y. (2015) Stochastic homogenization of a nonconvex HamiltonJacobi equation. Calc. Var. Partial Differential Equations 54, 1507-1524.

[5] Bourgeat A., Piatnitski A. (2004) Approximations of effective coefficients in stochastic homogenization. Annales de l'Institute Henri Poincare (B) Probability and Statistics, 40, 153-165.

[6] Bensoussan A., Blankenship G. (1988) Controlled diffusions in random medium. Stochastics 24.

[7] Bourgeat A., Mikelić A., Piatnitski A. (2003) On the double porosity model of a single phase flow in random media. Asymptotic Analysis, 34, 311-332.

[8] Bourgeat A., Mikelić A., Wright S. (1994) Stochastic two-scale convergence in the mean and applications. J.Reine Angew. Math. 456, 19-51.

[9] Caffarelli L.A., Souganidis P.E., Wang L. (2005) Homogenization of fully nonlinear, uniformly elliptic and parabolic partial differential equations in stationary ergodic media. Comm.Pure Appl. Math., Vol. LVIII, 0319-0361.

[10] Castell F. (2001) Homogenization of random semilinear PDEs. Probab. Theory related Fields, 121, $492-524$.

[11] Corrias L., Perthame B. (2006) Critical space for the parabolic-parabolic Keller-Segel model in $\mathbb{R}^{d}$. C.R. Acad. Sci. Paris, Ser. I 342, 745-750.

[12] Corrias L., Perthame B., Zaag H. (2004) $L^{p}$ and $L^{\infty}$ a priori estimates for some chemotaxis models and applications to the Cauchy problem, The Mechanism of the Spatio-Temporal Pattern Arising in Reaction Diffusion System, Kyoto, preprint available at http:// www.math.univparis13.fr/zaag/publications/CPZkyoto04.pdf.

[13] Corrias L., Perthame B., Zaag H. (2004) Global Solutions of Some Chemotaxis and Angiogenesis Systems in High Space Dimensions. Milan J. Math. 72, 1-28.

[14] Daley, D.J. and Vere-Jones D. An Introduction to the Theory of Point Processes. Volume II: General Theory and Structure, 2nd Edition. Springer, New York, 2008.

[15] Dal Maso G., Modica L. (1986) Nonlinear stochastic homogenization and ergodic theory. J. Reine Angewandte Math., 368, 28-42.

[16] Dal Maso G., Modica L. (1986) Nonlinear stochastic homogenization. Ann. Mat. Pure Appl., 144, 347-389.

[17] Evans, L.C. Partial Differential Equations. 2nd Edition, American Mathematical Society, 2010.

[18] Gajewski H., Zacharias, K. (1998) Global behaviour of a reaction-diffusion system modelling chemotaxis. Math. Nachr. 195, 77-114.

[19] Gray D.S., Tien J., and Chen C.S. (2003) Repositioning of cells by mechanotaxis on surfaces with micropatterned Young's modulus J Biomed Mater Res A, 66(3), 605-614.

[20] Heida, M. (2012) Stochastic homogenization of heat transfer in polycrystals with nonlinear contact conductivities. Asymptotic Analysis, 91, 1243-1264.

[21] Heida, M. (2011) An extension of the stochastic two-scale convergence method and application. Applicable Analysis, 72, 1-30.

[22] Hillen T., Potapov A. (2004) The one-dimensional chemotaxis model: global existence and asymptotic profile. Math. Meth. Appl. Sci., 27, 1783-1801.

[23] Horstmann D. (2003) From 1970 until present: the Keller-Segel model in chemotaxis and its consequences I. Jahresber. DMV, 105, 103-165.

[24] Horstmann D. (2004) From 1970 until present: the Keller-Segel model in chemotaxis and its consequences II. Jahresber. DMV, 106, 103-165.

[25] Horstmann D., Wang G. (2001) Blow-up in a chemotaxis model without symmetry assumptionns. European J. Appl. Math., 12, 159-177.

[26] Horstmann D., Winkler M. (2005) Boundedness vs blow-up in a chemotaxis system. J. Diff. Eq., 215, 52-107. 
[27] Jäger W., Luckhaus S. (1992) On explosions of solutions to a system of partial differential equations modelling chemotaxis. Trans. Amer. Math. Soc., 329, 819-824.

[28] Kallenberg, O. Foundations of Modern Probability, 2nd Edition. Springer, New York, 2002.

[29] Kechris, A. Classical Descriptive Set Theory. Springer, New York, 1994.

[30] Keller, E.F., Segel L.A. (1971) Model for chemotaxis. J. Theor. Biol. 30, 225-234.

[31] Kosygina E., Rezakhanlou F., Varadhan S.R.S. (2006) Stochastic homogenization of HamiltonJacobi-Bellman Equations. Commun. Pure Appl. Math., Vol. LIX, 1489-1521.

[32] Kozlov S.M. (1980) Averaging of random operators. Math. U.S.S.R.-Sbornik, 37, 167-180.

[33] Lions, J.L. Quelques Méthodes de Résolution des Problèmes aux Limites Non Linéaires, Dunod, 1969

[34] Lions P.-L., Souganidis P.E. (2005) Homogenization of "viscous" Hamilton-Jacobi equations in stationary ergodic media. Comm. Partial Diff. Equations, 30, 335-375.

[35] Lions P.-L., Souganidis P.E. (2010) Stochastic homogenization of Hamilton-Jacobi and "viscous" Hamilton-Jacobi equations with convex nonlinearity-revisited. Commun. Math. Sci., 8, 627-637.

[36] Mora X. (1983) Semilinear parabolic problems define semiflows on $C^{k}$ spaces. Trans. American Math. Society, 278, 21-55.

[37] Lukkassen D., Nguetseng G., Wall P. (2002) Two-scale convergence. Int. J Pure Appl. Math., 2, $35-86$

[38] Nagai T. (1995) Blow-up of radially symmetric solutions to a chemotaxis systems. Adv. Math. Sci. Appl.. 5, 581-601.

[39] Nagai T., Senba, Yoshida (1997) Application of the Trundinger-Moser inequality to a parabolic system of chemotaxis. Funkcial. Ekvac. Set. Internat., 40, 411-433.

[40] Nguetseng G. (1989) A general convergence result for a functional related to the theory of homogenization. SIAM J. Math. Anal., 20, 608-623.

[41] Osaki, K., Yagi, A. (2001) Finite dimensional attractor for one-dimensional Keller-Segel equations. Funkcialaj Ekvacioj 44, 441-469.

[42] Owhaldi, H. (2003) Approximation of effective conductivity of ergodic media by periodization. Probab. Theory Related Fields 125, 225-258.

[43] Papanicolaou G., Varadhan S.R.S. (1979) Boundary value problem with rapidly oscillating random coefficients. Collg. Math.Soc. Jámos Bolyai, 27, 835-873.

[44] Pazy, A. Semigroups of Linear Operators and Applications to Partial Differential Equations. Springer, New York, 1983.

[45] Perthame, B. Transport Equations in Biology. Birkhäuser, Basel, 2007.

[46] Yagi, A. (1997) Norm behaviour of solutions to a parabolic system of chemotaxis. Math. Japon. 45, 241-265.

[47] Winkler M. (2011) Finite-time blow-up in the higher-dimensional parabolic-parabolic Keller-Segel system. preprint, arXiv:1112.4156v1, 2011

[48] Winkler M. Aggregation vs. global diffusive behavior in the higher-dimensional KellerSegel model. J. Differential Equations, 248, 2889-2905.

[49] Wright S. (2001) On diffusion of a single-phase, slightly compressible fluid through a randomly fissured medium. Math. Meth. Appl. Sci., 24, 805-825.

[50] Zhikov, V.V., Kozlov O.A., Oleinik, O.A, Ngoan K.T. (1979) Averaging and G-convergence of differential operators. Russian Math. Surv., 34, 69-147.

[51] Zhikov, V.V., Kozlov O.A., Oleinik, O.A. Homogenization of Differential Operators and Integral Functionals. Springer, New York, 1994.

[52] Zhikov, V.V., Piatnitski, A.L. (2006) Homogenization of random singular structures and random measures. Izvestiya: Mathematics 70, 19-67. 